\title{
Imaginer le territoire de demain : le patrimoine comme levier de changement en EDD
}

\section{Imagine futur's of territory: Heritage as leverage of "change" in ES}

\section{Léna Jean-Yves ${ }^{1,2}$, Julien Marie-Pierre ${ }^{1,2}$, Calvet Anne ${ }^{3}$, Vergnolle Mainar Christine ${ }^{1,2}$, Chalmeau Raphaël ${ }^{2}$}

${ }^{1}$ Laboratoire GEODE UMR 5602 CNRS/UT2J - Labex DRIIHM/OHM haut Vicdessos, jean-yves.lena@univ-tls2.fr, marie-pierre.julien@univ-tlse2.fr, christine.vergnolle@univ-tlse2.fr

${ }^{2}$ ESPE Toulouse Midi-Pyrénées

${ }^{3}$ Rectorat Académie de Toulouse

Cette recherche a reçu le soutien du Labex DRIIHM ${ }^{1}$ et de l'Observatoire Hommes Milieux - Bassin Minier de Provence ${ }^{2}$ Remerciements à :

- L'école de Biver et les élèves des classes de CM1 et CM2, investis dans ce projet au long cours.

- Les enseignantes Marie-Christine Richard et Géraldine Pageon et toutes les personnes de l'école pour leur attention.

- Aux chercheurs de l'OHM-BM : Jacques Autran et Aurélie Arnaud, et à l'association « Graines d'Archi » de l'ENSAM, pour leur réelle implication tout au long de ce travail.

RÉSUMÉ. Cette recherche collaborative en éducation au développement durable (EDD) a été réalisée avec l'école élémentaire publique de Biver dans le cadre de l'Observatoire Hommes Milieux "Bassin Minier de Provence". Elle prend appui sur un modèle pédagogique d'arpentage du temps nommé " Tem Ter i 3 " qui se déroule en trois phases : présent, détour par le passé, projection dans le futur. La créativité est une des focales didactiques étudiée et la seconde concerne la prise en compte du "changement" en EDD. Le patrimoine et sa patrimonialisation ont servi de fil rouge à cette expérimentation dont les deux questions de recherche sont les suivantes: quels éléments de significations les élèves construisent-ils tout au long du dispositif ? Dans ce processus, quelles sont les formes de changements identifiables ? Les projections dans le futur, réalisées par les élèves, ont été analysées. Les principaux résultats concernent l'appréciation assez subtile par certains élèves de temporalités superposées et imbriquées les unes dans les autres. La sollicitation de l'imagination et de la créativité a permis aux élèves d'entrer dans des processus d'écriture et de réécriture, agissant dans de multiples dimensions : avec le réel et par le biais de ces requalifications d'espace.

ABSTRACT. This collaborative research in education for sustainable development (EDD) was implemented in the school of Biver (south-east of France), in territory of a Human Environment Observatory (HEO). It is based on a pedagogical model called " Tem Ter i 3 " which takes place in three phases (present, past detour, future projection) and an interdisciplinary context (based, here, on industrial heritage). The creativity is one of the didactic focal points studied, the second concerns the consideration of "change" in ESD. Our research questions are as follows: what elements of meaning do pupils construct throughout the device? and what are the identifiable forms of change? Projections in the future, carried out by pupils, were analyzed. The main results concern the rather subtle appreciation by some pupils of overlapping and interwoven temporalities. The solicitation of imagination and creativity allowed the pupils to enter into processes of writing and rewriting, acting in multiple dimensions: with reality and through these re-qualifications of space.

MOTS-CLÉS. éducation au développement durable, patrimoine industriel, changement, territoire, investigations multiréférentielles, architecture.

KEYWORDS. education for sustainable development, industrial heritage, change, territory, multi-referential investigations, architecture.

\footnotetext{
${ }^{1}$ Cet article s'inscrit dans le cadre du projet "Education au développement durable: changements et futurs possibles dans les territoires

" de I'OHM - BMP, financé par le Labex DRIIHM. http://www.driihm.fr

${ }^{2}$ https://www.ohm-provence.org
} 
"Ce petit non-espace-temps au cœur même du temps, contrairement au monde et à la culture où nous naissons, peut seulement être indiqué, mais ne peut pas être transmis ou hérité du passé ; chaque génération nouvelle ou même tout être humain nouveau en tant qu'il s'insère lui-même entre un passé infini et un futur infini, doit le découvrir et le frayer laborieusement à nouveau. » Hannah Arendt (1972)

"Nous ne savons ni où aller, ni comment habiter, ni avec qui cohabiter. Comment faire pour trouver une place? Comment nous orienter? » Bruno Latour (2017)

«Certes, je n'ai rien appris que je ne sois parti, ni enseigné autrui sans l'inviter à quitter son nid. [...] Qui ne bouge rien n'apprend rien » Michel Serres (1991)

"Qu'il fasse ou qu'il défasse, il n'importe ; il suffit qu'il change l'état des choses et tout changement est une action " JeanJacques Rousseau (1762)

\section{Introduction}

Cette étude a été réalisée sur la commune de Gardanne, avec l'école publique de Biver (cycle 3), dans le cadre de l'Observatoire Hommes-Milieux « Bassin Minier de Provence » (OHM-BMP), composante du programme Labex-DRIIHM CNRS. Ce territoire, marqué par cinq siècles d'extraction du lignite, a vu son dernier puit mettre définitivement fin à son activité extractive en 2003. Cette longue activité minière a reconfiguré le paysage et de nombreuses traces y sont encore présentes : descenderies, terrils, bâtiments industriels, chevalements, quartiers résidentiels ouvriers... (Autran et al., 2013). Mais ces traces ne sont pas toujours très lisibles pour les habitants et la mémoire du territoire oscille entre désintérêt et oubli. Cet espace est en forte recomposition économique et sociale. L'enjeu pour ce type de territoire est de rentrer dans un processus de résilience entendu comme une capacité à envisager un futur possible sans occulter le passé (Toubin et al., 2012).

Cette recherche collaborative prend appui sur un modèle d'arpentage du temps nommé « Tem Ter i 3 », Temporalités et Territoires ; innovation, investigation, imagination (Vergnolle et al., 2016) et se déroule en trois phases. Celui-ci débute par une observation-analyse du paysage actuel du lieu où vivent les élèves afin d'y repérer des enjeux au sein du socio-éco-système. Un détour par l'histoire est ensuite réalisé pour resituer ces enjeux dans une activité ancienne en lien avec le territoire. Puis les élèves se projettent vers le futur en élaborant des projets permettant ainsi d'adopter des postures d'acteurs et de citoyens de leur commune. Nous avons constaté dans nos précédentes mises en œuvre de ce modèle (Julien et al., 2014 ; Léna et al., 2016) des difficultés pour les élèves à s'engager dans le i de «imaginer » : ce travail est une réponse à ce constat récurent. Dans ce territoire, le patrimoine, choisi par l'équipe de mise en projet (enseignants, chercheurs et intervenants), semble constituer un point d'appui pertinent pour faire fonctionner le modèle dans un nouveau contexte.

Nous commencerons par mettre en évidence les nombreux liens entre l'éducation au développement durable et le terme «changement ». Puis nous exposerons la posture théorique au sein de l'éducation au développement durable qui nous parait la plus pertinente pour ce contexte : l'approche curriculaire et les investigations multiréférentielles d'enjeux. Enfin les termes «patrimoine » et «patrimonialisation » seront définis, ainsi que la notion de créativité au sein de notre cadre d'usage, le champ éducatif. 


\section{Cadre(s) théorique(s)}

\section{1. Éducation au développement durable et changement}

«Le corps qui traverse apprend certes un second monde, celui vers lequel il se dirige, où l'on parle une autre langue, mais il s'initie surtout à un troisième, par où il transite » (Serres, 1991). La métaphore du «métis » déclinée dans de multiples directions sert de pivot à Michel Serres pour aborder l'éducation et permet de l'associer à une odyssée du changement : changement de soi avant tout, par et avec l'autre, les autres ; changement de regard sur les objets qui nous entourent. Ces transformations individuelles ont nourri les interrogations sur les finalités de l'éducation à l'environnement. Selon Mappin et Johnson (2005), celle-ci a vu se succéder trois types de changement de finalité : les années 1970 prônaient le changement de comportement, les années 1980, le changement personnel par le biais de la compréhension des motivations et des attitudes personnelles qui guident les prises de décision. Puis dans les années 1990, l'objectif est devenu le changement des valeurs sociales et des systèmes pour sauvegarder la durabilité et la justice sociale. Les problématiques contemporaines de l'évolution rapide du climat et de la biodiversité ont réactualisé la question du changement dans nos sociétés, et dorénavant, climat et biodiversité sont l'une des signatures d'un changement d'ère géologique dénommée "Anthropocène ». Celle-ci résulte d'un faisceau convergeant d'activités humaines : des exploitations massives de ressources naturelles, des organisations sociales pas toujours équitables, et des systèmes économiques peu responsabilisés sur leurs effets. En d'autres termes, ce sont maintenant les géo-ingénieurs qui ont les clefs du «vaisseau terre » (Bonneuil et Fressoz, 2016). Dorénavant le changement est devenu une nécessité et le terme «transition» en est son mode opératoire.

L'éducation au développement durable (EDD) qui a succédé depuis une dizaine d'années à l'éducation à l'environnement est régulièrement sollicitée pour répondre aux attentes sociales. Elle s'empare également de ce questionnement. Deux objectifs principaux ont été mis en avant par 1'Unesco : « réorienter l'éducation et l'apprentissage de sorte que chacun ait la possibilité de se familiariser avec les connaissances, les compétences, les valeurs et les attitudes nécessaires pour avoir les moyens de contribuer au développement durable et accroître la place de l'éducation et de l'apprentissage dans tous les plans d'action, programmes et activités qui visent à promouvoir le développement durable » (Unesco, 2014). L'Éducation Nationale a transposé ces directives supranationales en circulaires éducatives, dont la dernière en date met particulièrement l'accent sur une citoyenneté active, via des mises en jeu de débats, des approches systémiques et complexes, un travail sur la prise en compte du futur dans les dispositifs proposés (MEN, circulaire $\mathrm{n}^{\circ}$ 2015-018 du 4-2-2015) ${ }^{3}$. Définissons plus précisément certaines dimensions associées au terme de changement dans la sphère éducative. Pour Abernot et Bedin (2015), le changement est évaluatif : "il consiste en une modification d'état (radicale ou progressive, régulière ou sporadique, prévisible ou surprenante) ou en une modification de rapport entre objets : positionnement, antériorité, inclusion, et surtout, de cause à effet, ferment de l'intelligence. L'évaluation consiste à établir une image d'état référé; dans le cas du changement, une modification accessible dans un système ». En prolongement, Janner-Raimondi et Tavignot (2016, p. 16) proposent que « la notion de changement ne saurait se faire sans lien avec une conception des rapports au monde et à autrui ». Par ailleurs deux régimes de changements peuvent être distingués selon certains auteurs. "Le niveau 1 renvoie à un changement dans la continuité, sans modifications du système ni recadrage. Pour le niveau 2, nous sommes autour du changement dans la discontinuité, la rupture, la transformation de la structure et de sa configuration, avec l'émergence de la nouveauté. [...] Les deux niveaux pouvant cohabiter parfois, dans

\footnotetext{
${ }^{3}$ Circulaire N²015-018 du 4-2-2015. Instruction relative au déploiement de l'éducation au développement durable dans l'ensemble des écoles et établissements scolaires pour la période 2015-2018.
} 
une temporalité donnée ou au cours d'une alternance dans le temps, c'est à dire dans un mouvement dialectique » (Saint-Jean et Seddaoui, 2013, p. 188). A ces deux régimes de changement, continuité et rupture, Janner Raimondi et Tavignot (2016) proposent une typologie à laquelle ils ont ajouté un troisième degré. Cette typologie est donc la suivante: (i) modification (continuité), (ii) métamorphose définie comme étant « une transformation externe modifiant l'apparence des choses, pouvant laisser croire à une mutation profonde » et (iii) transformation (rupture). Cette typologie nous servira de base pour l'analyse des travaux d'élèves (voir méthodologie). Une autre composante intéressante par rapport à cette recherche est proposée par Broussal (2017) qui associe le type de changement à la temporalité sous le terme de « trajectoire », ce qui « permet de dépasser la simple comparaison d'états ».

\subsection{Une approche curriculaire et multiréférentielle de l'EDD}

Comme le constatent Latour (2005) et Jolivet (2001, 2009), le développement durable (DD) bouleverse autant les cadres d'opérationnalisation que d'analyse des actions et implique de nombreux réajustements pour les situer de façon non réductrice. Les questions ou problématiques à caractère hybride sont constitutives du développement durable et l'usage de différents types de savoirs est nécessaire pour les traiter. Ils préconisent des résolutions orientées vers l'action obligeant à penser la science «en » société. La question des implications qui en résultent pour les contenus de l'EDD se trouve posée par Lange et Martinand (2010) qui conseillent une configuration de type curriculaire pour l'étudier. A partir du modèle de Ross (2000), Lange et Martinand (2010) définissent les trois pôles suivants : le pôle des actions éducatives de DD (AEDD) qui permettent aux jeunes de s'engager collectivement dans des actions, puis celui des contributions des disciplines et matières à l'étude d'enjeux du DD. Enfin, le troisième pôle concerne des investigations multi-référentielles (Ardoino, 1993) pour l'élucidation d'enjeux de développement et de durabilité. Ces mêmes auteurs soulignent la nécessité de caractériser la participation attendue et possible des élèves ainsi que la «préparation de ces futurs citoyens et acteurs sociaux à débattre, décider et agir ensemble en vue d'un développement durable selon une pratique effective de citoyenneté active ». Afin de caractériser la composante sociale et citoyenne dans l'EDD, Audigier (2011) propose la série de repères suivants pour les actions éducatives envisagées : 1) des situations convoquant une pluralité d'échelles spatiales, temporelles et sociales ; 2) un processus d'action et de décision ; 3) une prise en considération du futur ; 4) une alternance des dimensions individuelles et collectives ; 5) des confrontations à l'éthique et aux valeurs et 6) une combinaison de facteurs, c'est-à-dire la complexité. Cet ensemble invite à concevoir des dispositifs pédagogiques permettant la rencontre de différents types d'acteurs (experts, institutionnels, associatifs, témoins, acteurs économiques, ...) favorisant la multiplicité des points de vue, ou des cultures différentes pour aborder une question, un problème. En insistant sur les référencialités multiples portées par ces acteurs, Lange (2013) met en avant la pluralité des rationalités mises en jeu (techniques, éthiques, scientifiques, profanes, esthétiques ...) et les conséquences de possibles conflits entre ces rationalités ou ces systèmes référentiels, laissant apparaitre des zones d'ignorance, d'idéologies ou de croyance. Cette diversité, si elle est discutée, argumentée, située est le fondement de la construction de la démocratie, essentielle pour l'éducation selon Dewey (2011). Nul travail sur la construction de cet idéal démocratique n'est possible sans l'ancrer à une problématique dans un territoire local, facilitateur de sens pour les élèves, et permettant le jeu des temporalités. Ce fil conducteur est le patrimoine.

\subsection{L'enjeu de la patrimonialisation}

Une série de prises de conscience successives tout au long des deux derniers siècles a permis d'élargir progressivement le champ du patrimoine. En France, dès le $18^{\text {ème }}$ puis de façon amplifiée au $19^{\text {ème }}$, face à des destructions massives de bâtisses et de châteaux, de nombreuses personnalités (Mérimée, Stendhal, Hugo, Guizot...) alertent et incitent à la création d'un régime de protection spéciale pour les monuments nationaux puis dans un second temps pour certaines propriétés communales (Dorel-Ferré, 2007). La 
France va apprendre son histoire et se constituer comme propriétaire symbolique de ces monuments historiques (Dorel-Ferré, 2007). Plus tard, la convention UNESCO de 1972 définit le patrimoine comme l'héritage du passé dont nous bénéficions aujourd'hui et qu'il nous appartient de transmettre aux générations futures. Ce sera le leitmotiv du mouvement qui s'est intéressé plus particulièrement au patrimoine industriel, initialement sous l'angle d'ouvrages industriels en danger de dégradation (ponts, manufactures, pièces techniques...) puis ensuite aux savoir-faire, pour aboutir à la définition suivante : «Le patrimoine industriel est le champ de connaissance historique qui, à travers la collecte de témoignages oraux, matériels et immatériels, associe l'étude du bâti, le milieu géographique et humain, les processus techniques de la production, les conditions de travail, les savoir-faire, les rapports sociaux, les expressions culturelles, dans le but d'entrer dans l'étude des "sociétés fabricantes" quel que soit l'espace et le temps, depuis que ces sociétés existent » (Dorel-Ferré, 2007, p. 129). Plus tard, la convention-cadre européenne de Faro (2005) mettra l'accent sur le lien entre patrimoine et communautés humaines, et pose des principes de finalités (pourquoi) et de destinataires (pour qui) : «le patrimoine culturel constitue un ensemble de ressources hérités du passé que des personnes considèrent par-delà le régime de propriété des biens comme une expression de leurs valeurs, croyances, savoirs et traditions en continuelle évolution ». Ainsi ce sont tous les régimes de temporalités qui sont concernés.

Concernant l'éducation au patrimoine, Barthes (2017) note un glissement des intentions et des postures au fil des conventions: en 1972, les intentions éducatives sont centrées sur le « renforcement de l'attachement des peuples au patrimoine culturel et naturel » et posent un cadre supranational concernant l'éducation au patrimoine. En 2003, il est indiqué que «les États doivent mettre en place un développement de l'éducation en faveur du patrimoine », développant par ce fait, le patrimoine via l'action éducative. Puis en 2005, la convention précise que «le patrimoine est clairement présenté comme une ressource que les citoyens doivent faire émerger, et l'éducation est alors un moyen central mobilisé pour y parvenir. Ainsi, la fonction utilitariste de l'éducation au service de l'émergence d'une ressource est explicite ». Il est surprenant de constater à quel point patrimoine et DD ont de multiples points de convergence: initiation par une prise de conscience face à un danger, appui sur les sciences pour inventorier, classer, comprendre, travail sur les régimes d'historicité et invitation au final à une attention partagée au sujet du futur via l'usage commun du terme "générations futures ». Allant plus loin, Godard (1990) associe la définition du patrimoine à une recherche de prise de pouvoir sur l'avenir. Comme le territoire, « le patrimoine n'existe pas a priori » (Leniaud, 1992), c'est un construit historico-social. « Ni le patrimoine, ni les identités ne sont donnés a priori : ils sont indissociables de l'action de groupes localisés qui construisent la patrimonialisation de certaines ressources exclusives » (Nieddu et al., 2009). Le patrimoine est donc ce qui nous relie (Micoud, 2005), il est l'expression même de la relation sociale, ou tout au moins, d'un certain type de relations sociales (Nieddu et al., 2009). La patrimonialisation est le nom donné à ce processus par lequel « un collectif humain s'énonce comme tel par le travail de mise en collections de ce qui, de son passé, est pour lui gage d'avenir » (Micoud, 2004). Davallon (2014) propose « cinq gestes » nécessaires pour faire aboutir un processus de patrimonialisation : 1) un intérêt collectif pour un objet (ou un savoir) ; 2) une production de savoir sur l'objet ; 3) une déclaration du statut de patrimoine ; 4) organiser l'accès collectif à l'objet patrimonial puis enfin 5) le transmettre aux générations futures. Ce processus long, pluri-partenaires, par les multiples enjeux de significations rencontrées à toutes les échelles (individu, groupe, société) rattache pleinement le patrimoine au champ de la culture. Ce processus, tel que décrit précédemment, est également une des finalités des parcours d'éducation artistique et culturelle ${ }^{4}$.

\footnotetext{
${ }^{4}$ http://eduscol.education.fr/cid74945/le-parcours-d-education-artistique-et-culturelle.html
} 
Bien qu'une éducation «par » le patrimoine permette de travailler et puisse susciter une communauté de valeurs chez les élèves et une modification de leur regard sur leur environnement (Barthes et al., 2014) nous n'avons pas choisi cette focale pour ce travail, mais les préconisations de Tutiaux-Guillon (2017, p. 192) : " l'éducation au patrimoine suppose la mise en activité intellectuelle des élèves, leur implication dans des actions à leur échelle, le débat et la réflexion argumentée. Elle implique aussi un travail pluridisciplinaire, voire l'introduction de contenus qui échappent aux disciplines scolaires, qu'il s'agisse d'autres références savantes ou de savoirs vernaculaires ou de récits mémoriels ». La séquence pédagogique d'EDD analysée dans cet article utilise, donc, le fil rouge du patrimoine, mais cible davantage des compétences d'engagement citoyen, des mises en jeu multi scalaires d'espace et de temps à travers une projection vers le futur.

\subsection{Imaginer le futur du territoire : un enjeu éducatif intégrateur}

La projection vers le futur ne va cependant pas de soi, elle est particulièrement difficile pour les enfants et les adolescents. Penser le futur nécessite l'acquisition de compétences pour permettre aux jeunes d'identifier que plusieurs futurs sont possibles et ainsi apprendre à faire des choix en dépit de l'incertitude inhérente à cette réflexion. La démarche proposée vise, de la part des enfants, une meilleure compréhension de leur territoire et de ses dynamiques, afin de leur permettre de s'impliquer dans les débats le concernant. Cette question du futur est très peu prise en compte dans le domaine éducatif. Certains pays comme l'Australie (Bateman, 2012 ; Gough, 2010) et le Royaume Uni (Hicks et Holden, 2007) ont intégré cette réflexion du futur dans leurs curricula soit directement au travers des programmes soit au travers des « éducations à » la citoyenneté, l'environnement ou au DD. Seulement, il s'agit le plus souvent de références implicites, sans problématisation autour de cette question et sans articulation assez claire pour une réelle mise en œuvre (Gough, 1990). Les élèves n'ont pas l'habitude d'être mis dans ce type de contexte d'apprentissage et ont rarement, voire jamais, l'occasion de se projeter en « pensant par soi-même », éventuellement dans le futur ou a fortiori dans les futurs possibles (Julien et al., 2014 ; Julien et al., 2018). Cette phase d'imagination nécessite de la part des éducateurs de réelles attentions et plusieurs éléments structurant la mise en création d'un groupe d'élèves peuvent être repérés. Trois types de créativité sont mobilisés lorsque des personnes s'engagent dans l'agir selon Joas (1999); la créativité primaire pour la mise en œuvre de représentation ou l'enthousiasme, qui fait appel à l'imagination; la secondaire est la créativité pour la production rationnelle, la résolution de problèmes techniques, scientifiques, artistiques ...; et la troisième est la créativité intégrée qui associe «l'ouverture de l'expression de soi à la responsabilité du contrôle de soi ».

Sur le versant pédagogique, Hancock (1993) propose une activité dans laquelle il invite les participants à visualiser leur communauté idéale et à imaginer comment les personnes y vivent, y interagissent, y sont éduquées, y travaillent et y sont soignées. Ce n'est pas d'emblée sur la communauté que nous souhaitons initier cette phase créative du dispositif, mais à partir d'un lieu sur le territoire. Imaginer le futur d'un lieu permet dans ce cas également la mise en jeu des registres différenciés de technicités : le registre de la participation, celui de l'interprétation, celui de l'appropriation (jusqu'à la maîtrise), celui de la transgression (Martinand, 1994). Une fois les projets esquissés, une réflexion critique est nécessaire et peut être réalisée à partir des propositions de Pruneaux et Desjardins (2003) : Est-ce que mon projet contribue à polluer l'espace ? Est-ce que j'entre en contact avec des citoyens sur la place publique ? afin de critiquer, d'améliorer son projet et de l'inscrire dans une démarche citoyenne.

\section{Questions de recherche et déroulé}

Les questions de recherche suivantes nous ont servi de guide tout au long de ce travail : 
Concernant le patrimoine, quels éléments de signification les élèves construisent-ils tout au long du dispositif ? Dans ce processus quelles sont les formes de changement identifiables ?

Les élèves ont suivi une séquence-parcours constituée de onze étapes au cours desquelles deux fils conducteurs seront développés dans cette analyse : le patrimoine et le développement durable (figure 1).

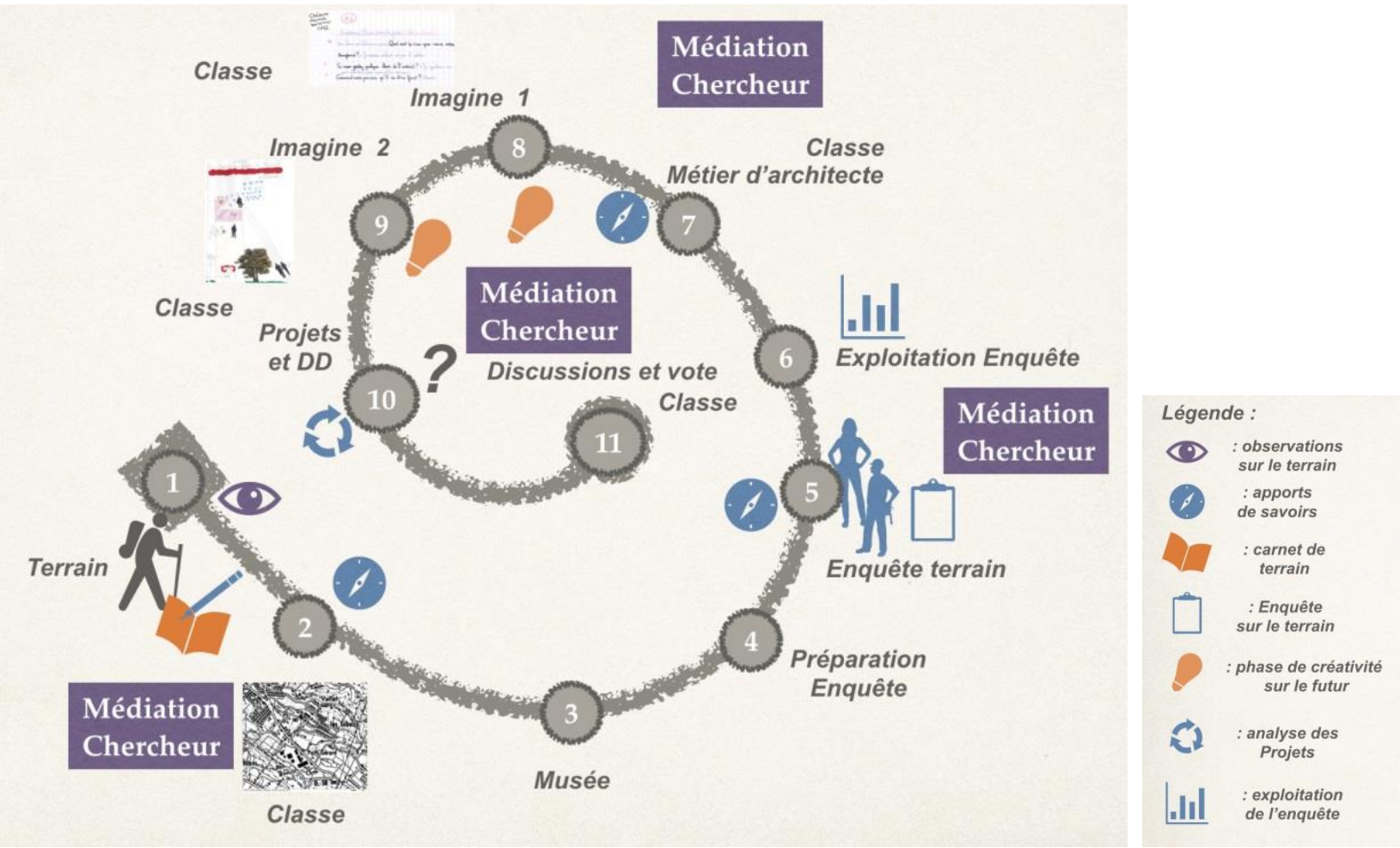

Figure 1. Séquence-parcours réalisée par les élèves et sa légende.

La première partie du parcours a permis l'étude des traces du passé sur le territoire (phases 1, 2 et 3), aussi bien lors de sortie de terrain (École, Sainte barbe, puits Biver, Terril, Ferme des Fabres, Statue du mineur, puits Gérard, Ferme en ruine, École) qu'au moment d'un travail en classe sur des cartes ou photographies aériennes anciennes datant de 1949. Cette première phase a permis de caractériser des traces du passé, de comprendre l'organisation du territoire à cette époque et de définir une première fois le terme « patrimoine ». Ce travail fut complété par une visite au musée de la mine de Mimet et sur le site de puits Gérard. Une enquête auprès des habitants de Gardanne sur la perception de leur territoire a été élaborée et réalisée par les élèves (phases 4, 5 et 6). Ensuite, lors de la phase 7, le métier d'architecte a été exposé et des rudiments de modalité de requalification d'un bâtiment ont été proposés sous forme de jeux.

En prolongement des sorties de terrain et du fil conducteur sur le «patrimoine », une première réflexion est proposée sur le lieu et la conception d'un projet d'aménagement qui se déroulera dans le futur (phase 8), puis dans un second temps une description sommaire du projet est demandée aux élèves intégrant des éléments suivants : «Nom de votre projet, quel lieu sur votre territoire, en quelle année se déroulera votre projet ? Que conservez-vous de l'endroit ? Quels sont les éléments du passé que vous souhaitez mettre en valeur ? Comment allez-vous faire ? » Ce cadre «pour imaginer» a abouti à une première esquisse. Une incitation à travailler en groupe a été formulée sans être obligatoire. Après un moment de présentation et d'échanges, une seconde esquisse a été réalisée en intégrant les différentes remarques.

Ensuite une phase (phase 10) de relecture des projets par les élèves à l'aide de la grille reportée dans le tableau 1, suivie d'une phase de possibles améliorations est réalisée avec les enseignants. Il n'y a pas eu 
d'apport spécifique au sujet du DD, afin d'éviter d'alourdir la séquence et ce sujet étant par ailleurs développé sur le territoire par des actions associatives ou institutionnelles.

\begin{tabular}{c|l|l}
\hline 1 & Prise en compte sociale & $\begin{array}{l}\text { Quelles personnes votre projet intéresse t-il ? (personnes âgées, } \\
\text { enfants, adolescents...) }\end{array}$ \\
\hline 2 & $\begin{array}{l}\text { Prise en compte des non- } \\
\text { humains }\end{array}$ & Quels types d'animaux et de végétaux sont présents dans votre projet ? \\
\hline 3 & Coût du projet (économique) ? & Combien coûte à peu près votre projet (1 maison $200000 €, \ldots) ?$ \\
\hline 4 & Effets en termes de pollution & $\begin{array}{l}\text { Quelles sont les pollutions (effets sur l'environnement) créées ou } \\
\text { produites par votre projet ? }\end{array}$ \\
\hline 5 & Place du patrimoine & Quels sont les éléments du passé mis en valeur dans votre projet ? \\
\hline
\end{tabular}

Tableau 1. Grille de questions pour analyser et critiquer son projet selon le DD et le passé (phase 10).

Les productions réalisées lors des étapes 8 et 9 (création d'un projet d'aménagement), 10 (analysecritique et amélioration selon le DD et prise en compte du passé - voir tableau 1 - et discussion des projets), 11 (présentation et choix des projets les plus significatifs) seront décrites, analysées et mises en perspective avec des éléments du parcours.

D'autre part, le rôle des chercheurs dans ce dispositif permet de travailler en réseau (Léna et al., 2016) et de varier les référentiels mobilisés lors de l'investigation, tant sur le plan des pratiques que sur le plan des types de savoirs mobilisés (tableau 2). 


\begin{tabular}{l|l|l|l} 
Étapes & Qui & Quoi & Temporalités \\
\hline $\mathbf{1}$ et $\mathbf{2}$ & $\begin{array}{l}\text { Jacques Autran, architecte géomaticien, } \\
\text { laboratoire ABC, école Nat. d'architecture - } \\
\text { ENSAM, Marseille }\end{array}$ & $\begin{array}{l}\text { Les traces du passé sur le terrain et sur } \\
\text { les cartes }\end{array}$ & Passé \\
\hline $\mathbf{4}$ et $\mathbf{5}$ & $\begin{array}{l}\text { Aurélie Arnaud, géographe urbaniste, } \\
\text { Laboratoire LIEU, Institut d'Urbanisme et } \\
\text { d'Aménagement Régional - Aix en Provence }\end{array}$ & $\begin{array}{l}\text { Enquête auprès des habitants } \\
\text { de Gardanne sur la perception de leur } \\
\text { environnement et le patrimoine }\end{array}$ & Présent \\
\hline $\mathbf{7}$ et $\mathbf{8}$ & $\begin{array}{l}\text { Camille Charpin, association Graine d'Archi, } \\
\text { ENSAM, Marseille }\end{array}$ & $\begin{array}{l}\text { Architecture/aménagement et initiation } \\
\text { des projets }\end{array}$ & Futur \\
\hline $\mathbf{1 0}$ et $\mathbf{1 1}$ & $\begin{array}{l}\text { Jacques Autran, architecte géomaticien, } \\
\text { laboratoire ABC, école Nat. d'architecture - } \\
\text { ENSAM, Marseille }\end{array}$ & $\begin{array}{l}\text { "Parole d'expert » pendant la discussion } \\
\text { puis le vote sur les projets des élèves }\end{array}$ & Futur/présent \\
\hline
\end{tabular}

Tableau 2. Les médiations effectuées par les chercheurs OHM durant la séquence-parcours.

\section{Corpus et méthodes d'analyses}

\subsection{Corpus}

La classe de CM1-CM2 a un effectif de 25 élèves. Le corpus analysé dans cet article provient des activités réalisées lors des phases 9,10 et 11 de la séquence, qui constituent donc les 3 dernières séances (cf. figure 1). Les projets réalisés par les élèves, sur papier, constituent le premier corpus. Ce sera dans la mesure du possible la seconde esquisse qui sera analysée, sauf si il n'en y a pas eue (ce qui est le cas pour 2 projets). Le second corpus est constitué par les réponses de élèves aux questions d'analyses de leur production (voir tableau 1) qui se sont matérialisés sur des «post it » de couleurs (chaque «post it » de couleur correspond à un type de question). Ces «post it » ont été photographiés et archivés. Le troisième corpus correspond à la présentation des projets (échanges enregistrés en vidéo) et au résultat du vote.

\subsection{Méthodes d'analyses}

L'ensemble des méthodes utilisées ci-dessous combine approche linguistique et approche anthropologique classique, de type ethnométhodologique défini par Garfinckel (2007). Nous situons notre ancrage analytique dans le courant pragmatiste (Mead, 2006; Dewey, 2011) adapté à des études d'analyses langagières par Bronckart (2007), qui stipule que les significations se construisent socialement (dans l'interaction), en relation avec les situations proposées et progressivement. Il propose plusieurs éléments clefs permettant une analyse possible : le langage est un médiateur entre les élèves et les processus d'apprentissage, les situations d'apprentissage permettent l'émergence de "textes » (toutes productions langagières) sur lesquels trois types d'indexation peuvent être réalisé : référentielle, communicationnelle, et culturelle. Bronckart (2007) envisage donc la médiation opérée par des signes, ouvrant la possibilité d'une sémiotisation. Ceci permet alors de faire le lien avec la vision peiricienne du patrimoine proposé par Tornatore. Celui-ci analyse le patrimoine comme un «opérateur singulier de signification sur la relation au passé, son caractère ou non d'héritage, avec ou sans testament, sa présence indicielle dans le présent, sur la continuité et la rupture, la permanence et le changement », et donc sur la transmission. Signification, relations indicielle, Tornatore (2010) indexe son analyse du patrimoine sur les 
éléments qui composent le système sémiotique de Peirce (1978). Sa théorie associe au signe trois pôles : le signe - l'objet - et l'interprétant qui se situerait entre l'objet et le signe. La production de sens émerge des interactions entre ces trois pôles. Peirce a dû distinguer trois types de signes, donc trois types de relations ou rapports entre l'objet et le signe, il les définit avec les termes suivants : 1) l'icône partage des éléments en commun avec l'objet, 2) l'indice est lié de façon logique avec l'objet 3) le symbole est lié de façon conventionnel avec l'objet. Cette position théorique a une conséquente immédiate, elle souligne le fait que le langage n'est pas figé et les significations ne peuvent se construire que collectivement par le jeu du dialogue. Ainsi donc chaque interprétation d'un signe suppose de «l'investir de significations nouvelles, parce que le signifiant ne véhicule pas d'un esprit à l'autre un concept sans que le jeu de l'interprétant transforme ce dernier, l'individu impliqué dans une activité discursive opère une action créatrice» (Pesce, 2008).

C'est bien dans le mouvement des échanges, le jeu des interprétants qu'une production de significations sera possible par les élèves.

Concrètement, la figure 2 illustre les différentes étapes suivies et les focales effectuées sur les productions des élèves; la première focale concerne les modes de représentations des élèves, les formes dessinées et leur dispositions dans l'espace (macro-analyse graphique); la seconde focale concerne le lexique utilisé par les élèves dans leur dessins; enfin la troisième focale se concentre sur les points de détail dessinés des projets (micro-analyse graphique). L'ensemble des signes sont relevés, et triés, selon ces focales, comme le montre le tableau 3. Ensuite deux traitements interprétatifs sont réalisés : le premier via une indexqtion permet de faire émerger éléments référentielles (Bronckart (2007) et le second abouti à catégoriser les types de changements proposés par les élèves (modification, métamorphose, transformation) est réalisée à partir de Janner-Raimondi et Tavignot (2016).

Les grilles de créativité de Joas (1999) et les registres de technicités de Martinand (1994) ont complété les interprétations développées dans la discussion.

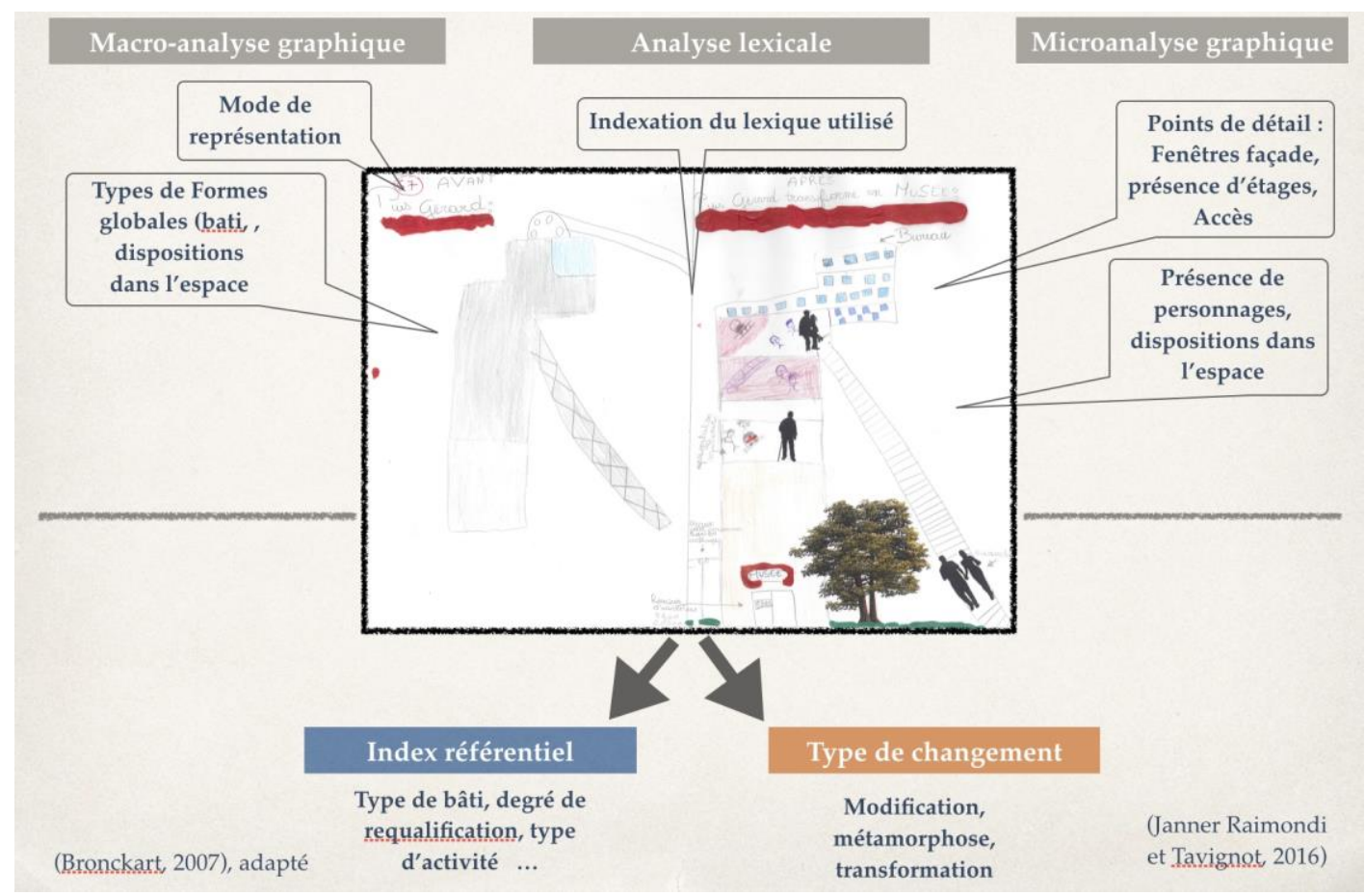

Figure 2. Éléments et étapes de l'analyse sémiotique et sémantique, graphique et lexicale des projets réalisés lors des étape 7 et 8. 


\begin{tabular}{|c|c|c|c|}
\hline Analyse macro-graphique & Analyse lexicales & Analyses micro-graphiques & Exemples de projets \\
\hline $\begin{array}{l}\text { Identité du bâtiment reconnaissable } \\
\text { (ex : puit Gérard). } \\
\rightarrow \text { Bâtiment déjà existant modifié }\end{array}$ & $\begin{array}{l}\text { " transformé », } \\
\text { " représentation des } \\
\text { mineurs ", " ascenseur pour } \\
\text { handicapé », " Horaires } \\
\text { d'ouvertures ». } \\
\rightarrow \text { Soin aux détails de } \\
\text { fonctionnement }\end{array}$ & $\begin{array}{l}\text { Détails sur l'organisations des } \\
\text { espaces intérieurs (fonctions } \\
\text { des pièces identifiables et } \\
\text { symbolisées), micro - détails : } \\
\text { des personnages sont } \\
\text { représentés en situation. } \\
\rightarrow \text { Soin aux aménagements } \\
\text { intérieurs }\end{array}$ & $\begin{array}{l}\text { Musée de la mine } \\
\text { Type de Changement : } \rightarrow \\
\text { Métamorphose (bâtiment } \\
\text { conservé réaménagé et } \\
\text { fonction réorientée) }\end{array}$ \\
\hline $\begin{array}{l}\rightarrow \text { Nouveaux bâtiments et nouvelle } \\
\text { physionomie urbaine. }\end{array}$ & $\begin{array}{l}\text { "la tour de Biver, } 75 \mathrm{~m} \text {, la } \\
\text { halle, Carrefour market, } \\
\text { Pharmacie, le rimbaud ". } \\
\rightarrow \text { des noms qui combinent } \\
\text { l'ancien et le nouveau ; } \\
\text { l'échelle du lexique : la ville. }\end{array}$ & $\begin{array}{l}\text { Panneaux signalétiques } \\
\text { (Pharmacie, Carrefour } \\
\text { Market). } \\
\text { Détail de l'entrée de la } \\
\text { piscine. } \\
\text { Larges espaces autours des } \\
\text { constructions. } \\
\rightarrow \text { Soin aux espaces et aux } \\
\text { fonctions. }\end{array}$ & $\begin{array}{l}\text { Gardanne Biver dans le } \\
\text { futur } \\
\text { Type de Changement : } \rightarrow \\
\text { Transformation (nouveaux } \\
\text { bâtiments et nouvelles } \\
\text { dispositions et fonctions) }\end{array}$ \\
\hline $\begin{array}{l}\text { L'usine Altéo fabriquant l'aluminium est } \\
\text { clairement reconnaissable (aspect } \\
\text { extérieur conservé). Mais son } \\
\text { organisation intérieure est } \\
\text { complètement repensée avec de } \\
\text { nouvelles finalités tout en conservant le } \\
\text { lien historique avec l'aluminium. }\end{array}$ & $\begin{array}{l}\text { "Détail du projet, cuve } \\
\text { aquarium, un scilo sur l'étude } \\
\text { de l'alumine, un scilo marché } \\
\text { et jeux vidéos, pharmacie" } \\
\rightarrow \text { des noms qui combinent } \\
\text { l'ancien et le nouveau; } \\
\text { l'échelle du lexique : la ville }\end{array}$ & $\begin{array}{l}\text { Grand nombre de fenêtres, } \\
\text { toute à la même forme: } \\
\text { carré. Détail de l'aquarium, } \\
\text { avec dessin de poissons. } \\
\text { Une personne à la base du } \\
\text { dessin. } \\
\rightarrow \text { souci de la perception de } \\
\text { l'échelle }\end{array}$ & $\begin{array}{l}\text { L'alumine } \\
\text { Type de Changement : } \rightarrow \\
\text { Métamorphose (bâtiment } \\
\text { conservé réaménagé et } \\
\text { fonctions réorientées) }\end{array}$ \\
\hline
\end{tabular}

Tableau 3. Exemple de construction des index référentiels et des types de changements à partir des signes relevés dans les productions. Les groupes de mots suivants les $\rightarrow$ signalent un début d'interprétation.

\section{Résultats \& analyses}

\subsection{Les Projets}

\subsubsection{Description générale et types de projets}

Au total, les élèves ont produit 16 projets dont leurs horizons de réalisation s'étalent de 2020 à 2045 . De nombreux projets ont pour ancrage le secteur des loisirs (camping « des loups », parc aquatique, 
bâtiment omnisport, parc d'attraction «chadeux of évil », pistes de motos et vélos) ou le commerce, intégré sous forme de «complexe commercial » (« Restau Europstar » comprenant un « micromania », un «Go sport » et un « centre commercial»). Quatre projets ont une finalité culturelle sous forme de musées («Musée de la mine », «musée St Jean »). Un projet de reconversion complexe de l'usine Altéo, baptisé «alumine», puis deux projets atypiques qui ont souhaité réorganiser toute la ville et proposer une nouvelle urbanité (« on rase tout et on recommence »).

L'analyse sémantique des noms des projets révèle qu'une majeure partie désigne l'objet lui-même qui sera réalisé (13 sur 16) dont certains sont repris des noms déjà existants (noms d'enseigne, tel « Micromania »), un projet n'a pas souhaité donné un nom mais une devise « je rase et je reconstruis »synonyme sans doute d'une nouveauté radicale y compris dans le nouveau nom de la ville à imaginer puis à construire. Un seul projet s'affuble d'un nom propre «Europstar» associé à un projet de restaurant. Enfin un projet se singularise par un nom polysémique « l'alumine», dont rien dans le titre n'annonce une orientation en termes de types de lieux. Donc au final les noms des projets sont porteurs de peu de nouveauté.

La richesse sémiotique ne se réduit pas seulement au langage verbal, et de multiples éléments de significations sont présents dans la partie graphique des dessins qui se révèle très riche (voir Tableau synthétique en annexe 2). Les projets sont souvent situés dans un espace plus vaste que l'objet présenté, avec notamment une prise en compte du sous-sol, et une insertion sur site (voir figure 3, musée du terril et annexe, piscine du terril, le projet alumine). D'un projet émane une signature graphique singulière et très aboutie. Il s'agit du «Musée de la mine sur le terril » (voir figure 3). Celle-ci est constituée d'une alternance de bandes de couleur marron verticales dans la partie souterraine, et horizontales dans la partie aérienne. Elle révèle une maitrise de la symbolique graphique avancée et correspond à une pratique actuelle des grands projets d'architecture dans l'espace urbain de créer des « signature du lieu » ou un design de paysage urbain. L'ascenseur extérieur de ce même projet est également symptomatique d'un processus complexe concernant les solutions techniques envisagées et adaptées. Au regard de contraintes de hauteur ou du type de bâtiment et de la volonté de conserver une relation au passé (présence des câbles, de la fonction du bâtiment), une solution originale est proposée.

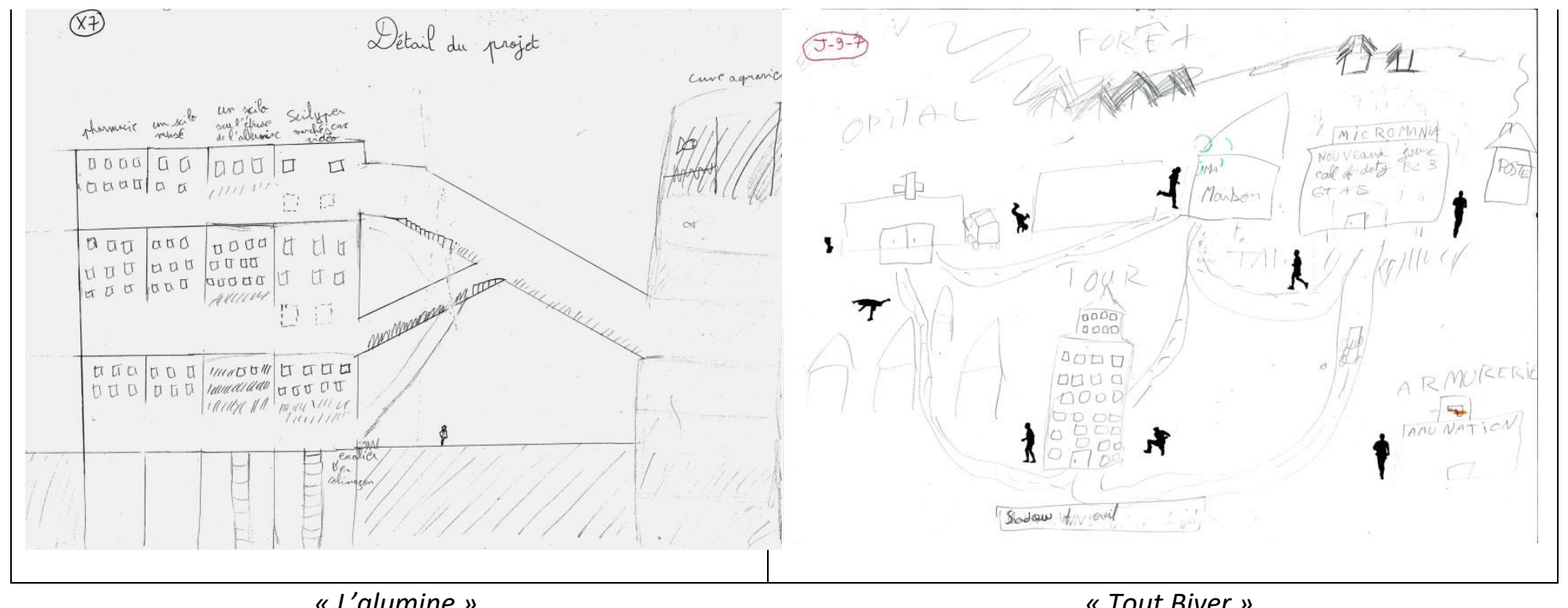




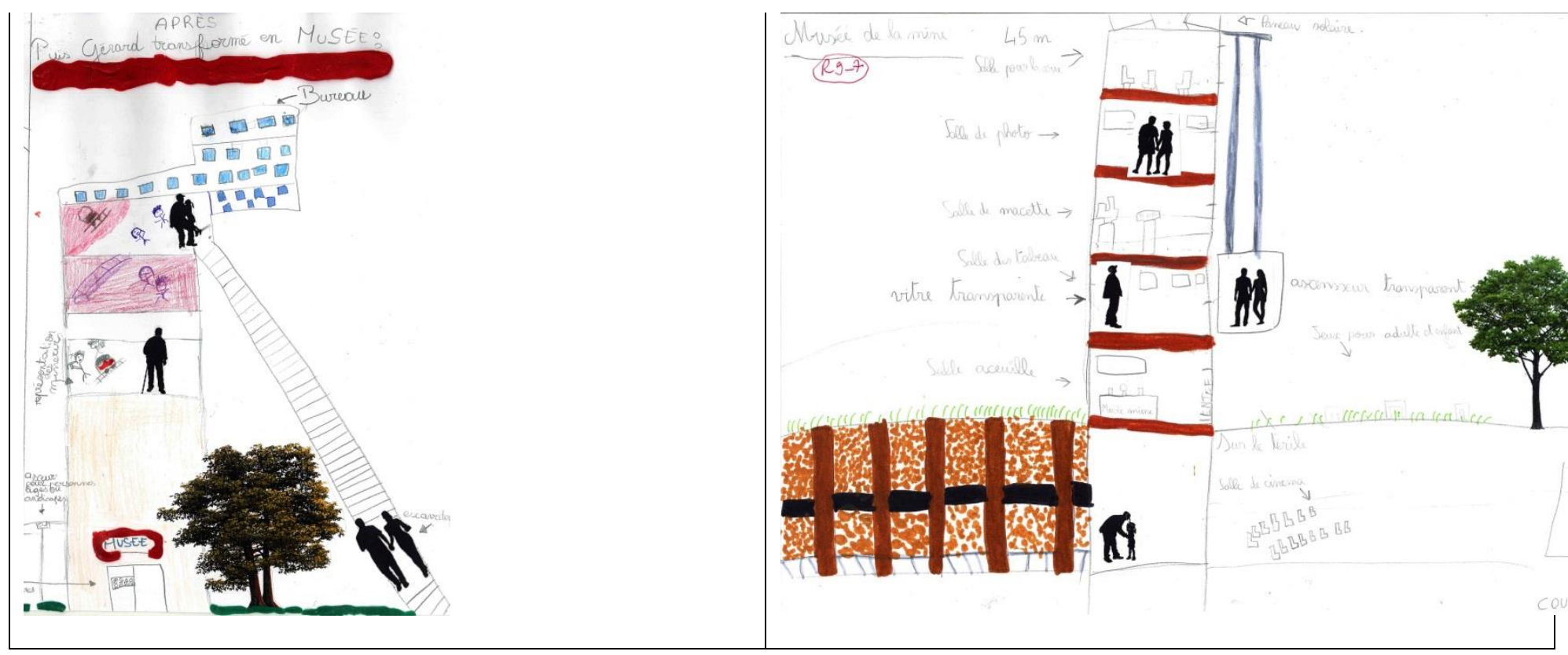

"Musée de la mine à puits Gérard »

"Musée de la mine sur le terril »

Figure 3. Exemples de projets imaginés par les élèves.

Sur l'ensemble des projets des figurations de mises en jeu du temps sont proposées par différents procédés : 1) légendes ou panonceaux qui précisent des horaires, 2) des personnages sont présents dans la moitié des dessins, ceux-ci sont positionnés de telles façons qu'ils représentent des personnes en « situation » d'action dans le bâtiment, 3) juxtaposition de dessins façon BD qui indique pour un même lieu une séquence de fonction possible (dans la piscine: bassin, sauna, salles de massage, salle de détente ...). Le camping est quasiment prêt à fonctionner avec des indications, et des éléments pratiques proposés (signalétique, organisation des espaces). Vu le délai de réalisation dont ont disposé les groupes pour fabriquer ces esquisses, ces indices graphiques de représentations signifient que le projet et son fonctionnement sont pensés de façon synergique.

Sur un autre registre il est intéressant de noter que diverses formes de travail en groupe ont été mobilisées; du travail distribué et organisé à l'exercice solitaire une vaste palette de modalités a pu se faire. Le projet «Restau Europstar » (code LTBH, annexe 2) a réuni un groupe de quatre élèves au sein duquel plusieurs sous projets ont pu se développer de façon coordonnée, ce qui a donné quatre esquisses pour un seul projet. Le projet « Chadeux of evil » a réuni deux élèves qui n'ont pu se mettre d'accord sur un lieu commun et ont donc décidé de faire un projet similaire sur deux lieux différents (l'un en forêt et l'autre en ville).

\subsubsection{Les types de projets et les lieux / la signature d'un rapport à l'espace}

Tous les projets n'ont pas proposé un lien direct avec le patrimoine, puisque la consigne de départ a présenté le patrimoine comme possible mais pas obligatoire : $28 \%$ des projets n'ont donc aucun lien avec le patrimoine, $47 \%$ des projets concernent un bâtiment ou un site industriel, liés à l'activité minière. Dans la majeure partie, le bâti a été conservé et requalifié (en termes de fonction et d'usage) dans le secteur des loisirs (installations sportives) et celui de la culture-mémoire (musées) comme en témoigne la figure 4. 


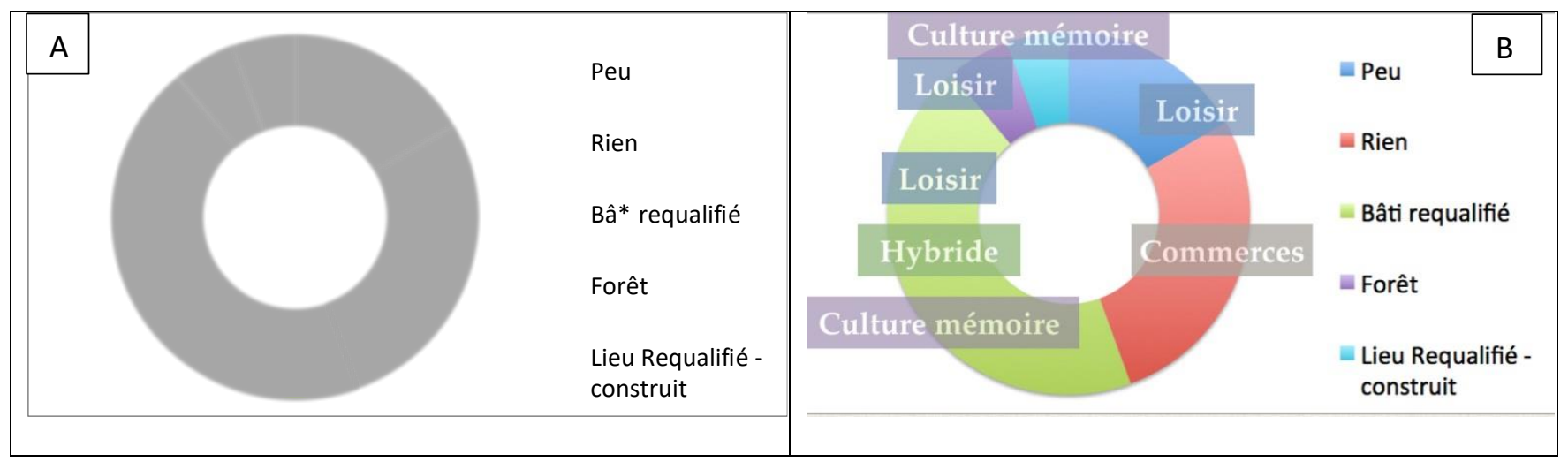

Figure 4. Proportion de projets selon type de patrimoine $(A)$, proportion de type de projets selon type de patrimoine $(B)$.

Il est intéressant de relever que les projets liés au commerce présentent une certaine ambiguïté. Ils sont pour la plupart associés au projet « restau Europstar » situé sur le site d'Altéo, mais dans leurs esquisses les élèves ne mentionnent pas le site et il semble «hors-sol ». Est-ce lié à la genèse du projet ou à la qualité du commerce qui peut être en tout lieu, c'est-à-dire que peu importe le lieu où le site ? Il est à noter également que les sites de loisirs se répartissent dans tous les lieux du quartier Biver de la ville de Gardanne (le terril, Puits Gérard, la ferme des Fabres, la ferme en ruine, la forêt).

\subsubsection{La problématique du changement / la signature d'un rapport au temps}

Cette dimension du changement est présente dans les projets, de façon plus indicielle, pour reprendre une terminologie de Peirce (1978). Tous les projets proposent un changement d'un bâti ou d'un lieu ou les deux, soit en requalification partielle, soit en requalification totale comme c'est le cas du projet «alumine » (figure 5). Pour ce projet il s'agit de reconfigurer la fonction du bâtiment en laboratoire d'étude sur les effets de l'aluminium. Chaque étage représenté est dédié à une part du projet : un centre de recherche sur l'aluminium (impacts sur la santé humaine), une pharmacie pour soigner les problèmes engendrés par ce même aluminium, des logements pour les étudiants présents dans le centre de recherche, quelques commerces. Le projet conserve la physionomie d'un des bâtiments du site mais le requalifie intégralement. Cette reconversion, correspond à une transformation tout en conservant le thème de l'aluminium et en l'orientant vers le soin, signale une double dimension temporelle : une continuité et une rupture.

Quatre projets parmi les seize se sont clairement démarqués de l'ensemble des projets et ont symboliquement traité la dimension du changement. Le projet du musée à puits Gérard, a présenté son projet dans deux phases de temps bien distinctes : «avant» et «après ». Certains éléments sont conservés (le puits en lui-même) et une réaffectation du bâtiment en musée est proposée (voir l'annexe 3). Cette proposition évoque le jeu des sept différences, ou deux présentations très similaires ne varient que par sept détails et invite à la comparaison et à la réflexion. Enfin, trois projets ont opéré des changements radicaux : deux concernent Biver et Gardanne qui seraient rasés puis reconstruits avec des services plus identifiables (hôpital, poste, commerces), puis un dernier «Chadeux of evil», un projet de «parc d'attraction » qui sortira de terre, sans rien conserver du passé, peu importe le lieu, soit sur un emplacement de forêt soit de ville. Ces projets sans rapport avec l'esprit du lieu signalent une «transformation » selon les catégories de Janner-Raimondi et Tavignot (2016). 


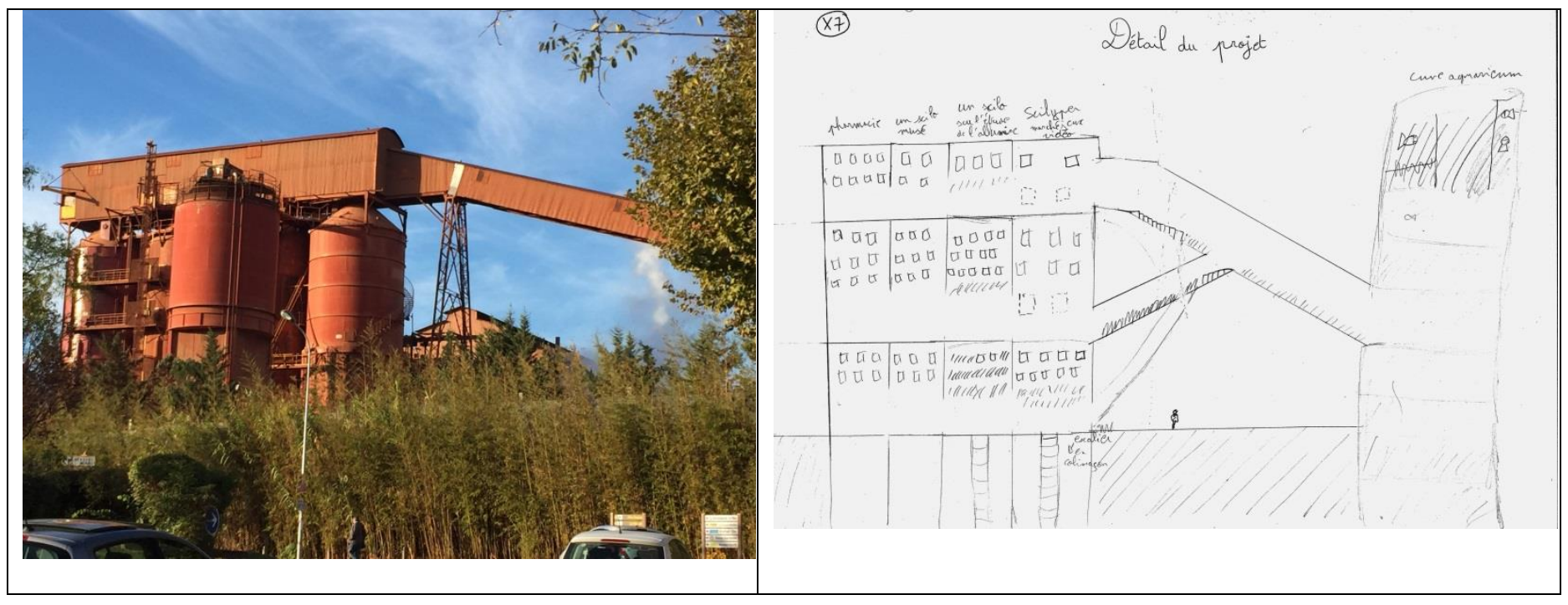

Figure 5. Les changements dans les projets : exemple «l'alumine », à partir de l'usine Altéo de Gardanne.

\begin{tabular}{|l|c|c|c|}
\hline Type de changement & Modification & Métamorphose & Transformation \\
\hline $\begin{array}{l}\text { Exemples de projets et } \\
\text { lieux }\end{array}$ & Camping (Forêt Biver) & $\begin{array}{c}\text { Musées de la mine (terril } \\
\text { de puits Biver, Puits } \\
\text { Gérard) }\end{array}$ & $\begin{array}{c}\text { Parc d'attraction } \\
\text { (Gardanne) }\end{array}$ \\
\hline Nombre de projets & 2 & 10 & 4 \\
\hline
\end{tabular}

Tableau 4. Nombre de projets par type de changements

\subsection{La phase « critique des projets » puis la discussion pour choisir trois projets}

Au cours de cette phase les annotations critiques ont été catégorisées et comptabilisées dans le tableau ci-dessous.

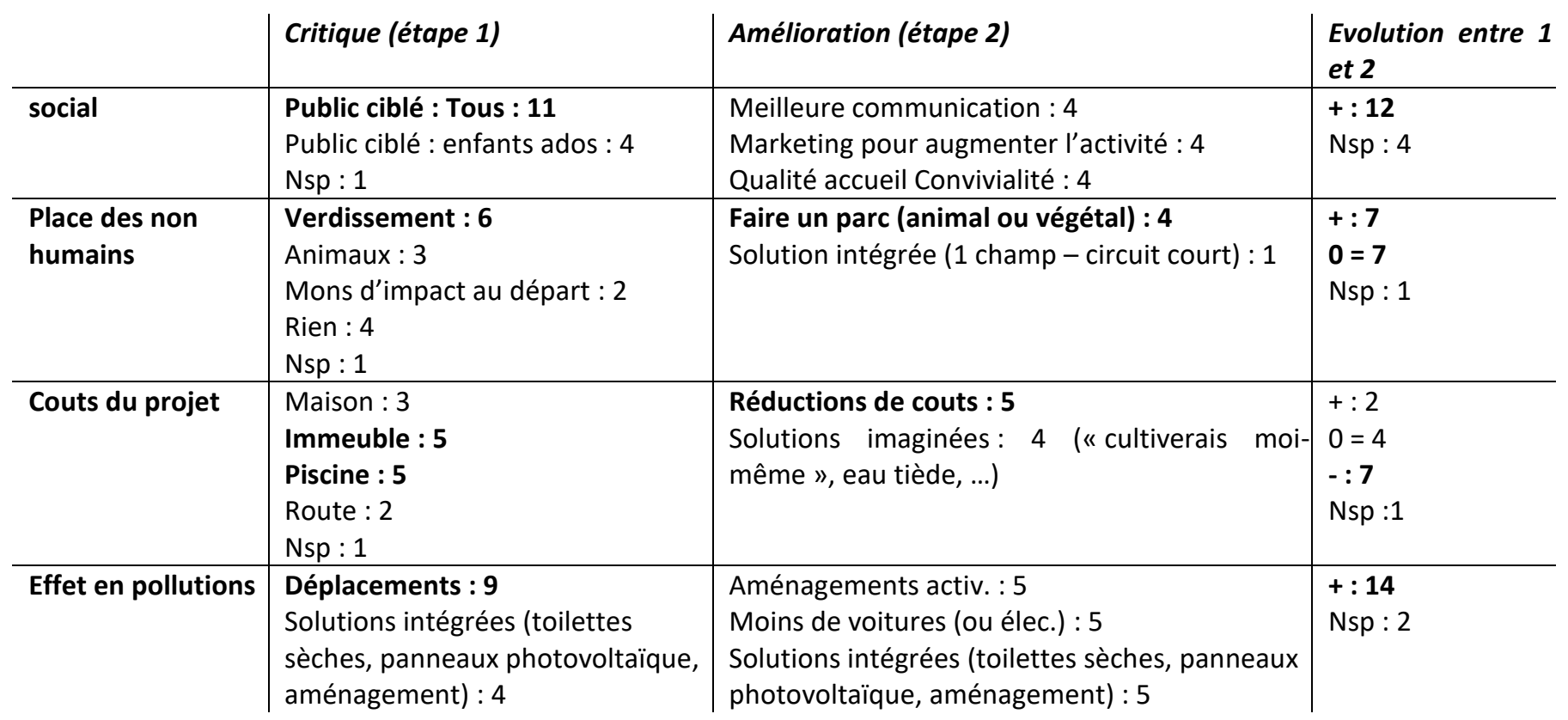


Forêt : 5

Bâtiment industriel : 5

Divers (Statue) : 1

Site : 1

Tableau 5. Annotations des élèves lors de la critique puis lors de l'amélioration des projets. (Légende dernière colonne : elle comptabilise pour chaque projet les différences entre étape 1 et 2, le signe « + » compte les améliorations, le signe « - » les non prises en compte, et le signe « 0 »l'absence d'évolution).

Tous secteurs confondus, cette phase a engagé tous les élèves vers une évolution notable de leur projet avec dans un premier temps une relecture de leur travail avec d'autres « lunettes » que celles utilisées habituellement, puis une réflexion afin de mieux prendre en compte certaines composantes (social, relation au passé, et effet des pollutions). Le domaine «non humain », c'est-à-dire les relations envers les animaux et les végétaux, révèle un rapport asymétrique de domestication (la plante comme décor, non humains absents, le parc comme solution d'amélioration), soit un modèle courant de relation à l'animal (Descola, 2005) sans doute amplifié par le modèle de vie urbaine et par les thématiques des projets. Malgré tout, une prise en compte du DD de façon intégrée semble en acte.

La présentation des projets a permis aux élèves d'expliciter davantage leur choix, et de donner des détails supplémentaires quant aux possibilités de leurs projets et de leur prise en compte des différents éléments souhaités. Lors du débat qui a succédé, six projets sur seize ont été discutés, voir défendus et presque tous les élèves se sont exprimés au cours de ces échanges. Le vote a porté sur une double qualité : choisir un projet qui traite du patrimoine et prenne en compte le DD. Les élèves ont voté majoritairement pour le projet «camping des loups » et à la dimension patrimoine industriel abordé tout le long de la séquence, ils ont répondu... patrimoine naturel! Trois projets ont récolté 20 voix ce qui représente $80 \%$ des voix de la classe, reflet d'un choix homogène (tableau 6).

\begin{tabular}{|l|c|c|c|}
\hline Nom du projet & Camping des loups & Musée de la mine & L'alumine \\
\hline Localisation & Forêt Biver & terril de puits Biver & Site Altéo - Gardanne \\
\hline Nombre de voix & 9 & 6 & 5 \\
\hline
\end{tabular}

Tableau 6. Résultat du vote à l'issue de la discussion des projets.

\section{Discussion}

\subsection{Le fil du patrimoine, de la patrimonialisation et du travail des liens via la démarche multiréférentielle}

Le fil du patrimoine a permis de travailler avec les élèves sur ce qui marque le territoire de façon intentionnelle afin de les aider à passer de la pratique d'un lieu à sa signification (Vergnolle Mainar et al, 2012). Les trois grands moments de la démarche adoptée ont permis une approche itérative au sujet du patrimoine via l'arpentage du temps. Les investigations multiréférentielles des enjeux engagent les jeunes dans une construction du terme en constante évolution et invitent à diverses modalités d'appropriation. Les traces du passé, discrètes mais encore actives dans leur paysage ont abouti à une nécessaire première définition: «un élément du passé que nous choisissons de conserver ». L'enquête "perception à Gardanne », qui a suivi, a permis une confrontation aux visions des autres habitants qui associent le terme patrimoine aux «monuments ». Celle-ci est une formule brève qui conserve en elle intacte la dimension 
«propriété symbolique », sur laquelle s'est bâtie l'histoire de l'Histoire de France, depuis le 19ème siècle. Les projets imaginés par les élèves ont permis de tisser de nouveaux liens avec le terme patrimoine : les notions de choix (quels lieux, quels objets) et de mises en valeur (musée ou autres) mais aussi de confronter cette notion avec leurs propres désirs ou leurs références socio-économiques (loisirs, commerce). Les moments collectifs de présentation, de discussions et de vote ont permis de mettre en évidence des facettes simplement évoquées dans leur parcours mais peu développées, et l'issue du vote révéla une composante importante, le patrimoine naturel.

La démarche d'investigation multiréférentielle utilisée dans cette recherche, induit par les rencontres au cours du parcours certaines références plutôt que d'autres (dans notre cas : architectes, patrimoine industriel, activités autour du charbon) mais n'est jamais complètement aboutie (Lange (2013); c'est une de ces caractéristiques et limites. Cette démarche laisse une part d'orientation forte aux élèves, n'aboutissant pas nécessairement à l'horizon envisagé initialement par les adultes. L'attention de deux groupes vers l'usine Altéo en est une illustration. Cette usine, au cœur de la ville, semble faire problème pour certains élèves et leur proposition est de remplacer sa fonction industrielle par des activités de loisirs ou de recherche. Un parcours de ce type est propice à la mise en résonance de significations multiples rencontrées tout au long du «chemin » parcouru. Réfléchir collectivement à un projet, l'esquisser, le dessiner, le critiquer (voir tableau 4), le présenter, le voter, c'est vivre chaque étape d'un processus qui dans la société civile est long, complexe, constellé d'experts et d'institutions (Davallon, 2014). L'ensemble de ce vécu est assez proche de la vision de la patrimonialisation de Tornatore (2010) : « une façon de faire du lien entre les bâtiments, les objets, autant de constructions humaines donc une certaine forme de matérialité et un sentiment partagé, (dans lequel l'affectif ou l'émotion vont jouer un rôle central) qui va se muer en valeur » et cela leur permet « donc de s'engager dans une démarche politique ».

Une autre caractéristique symbolique du patrimoine, c'est d'introduire une continuité dans le temps à l'intérieur de la société. Micoud (2005) insiste sur la qualité et la conscience des liens que nous sommes capables de tisser avec le territoire, les objets et le temps et évoque la nécessaire réappropriation des objets en question (dans ce cas le patrimoine de proximité). Ceci passe par des phases de re-sémantisation. Le dispositif élaboré, notamment sa partie «projet imaginé » vécue par les élèves, favorise des phases d'écritures et de réécritures, d'invention et de mise à distance, s'engage donc particulièrement dans cette voie.

\subsection{Figurations du changement et relation aux temporalités}

A partir de sites du territoire, les projets ont proposé des modifications voire des transformations de bâtiments ou des aménagements de lieux. Cette requalification porte en elle différents types de changements. Un type de changement au regard de la grille utilisée est majoritairement proposé par les élèves, il s'agit du changement de type «métamorphose » : 1- 10 projets sur 16 proposent un changement de type métamorphose, où souvent une part du bâti est conservée et un nouvel usage est imaginé (musées, piscine, salle de sport), à l'image de la requalification de bâtiments industriels longtemps restés en friche et transformés en lieux culturels ou de loisirs. Dans ces projets, le lien avec le passé est conservé, voire dans certains cas valorisés, comme c'est le cas des projets de musées de la mine. 2- Les quatre projets proposant un changement avec «transformation » signalent une rupture forte avec le passé, ou le présent ; dans ces cas, tout est reconstruit, rien n'est conservé et l'esprit du lien est profondément modifié. Les projets proposés sont souvent sans ancrage territorial précis et liés à une activité commerciale. 3- Les deux projets correspondant à un type de changement avec «modification» signalent une plus grande continuité avec le présent ; conservation du site ou d'un bâtiment comme c'est le cas de la conversion de terrain agricole ou d'abords non-construits, en zone sportive ou de loisir comme pour le projet de camping. 
La réalisation des projets, puis les différents moments collectifs de confrontations, permettent aux élèves de valider des choix, de les mettre en discussion, voir de les peaufiner. Ceci abouti à des propositions très variées au sujet des temporalités et du changement, révélant une perception des continuités et des ruptures dans le territoire plus nuancée qu'on ne le supposait au départ. En effet, Bergson (1969), lors de ses conférences sur la perception du changement, évoque une notion paradoxale et pourtant qui trouve un écho dans cette forme invisible présente dans ce projet du camping. Il associe changement à «l'indivisibilité»d'une action; «ce n'est pas seulement notre passé à nous qui se conserve, c'est le passé de n'importe quel changement, pourvu toutefois que nous ayons bien affaire à un changement unique et, par là même, indivisible : la conservation du passé dans le présent n'est pas autre chose que l'indivisibilité du changement » (Bergson, 1969, p. 35). Effectivement les temporalités étudiées apparaissent ensemble tissées entre elles comme « les historiens le savent bien, depuis Fernand Braudel : il y a plusieurs temps superposés dans un moment historique, et c'est ce feuilleté chronologique, cette stratigraphie souvent inaperçue » (Bougnoux et Debray, 2013) qu'il nous convient de mettre en chantier avec les élèves. Le projet «l'alumine », par sa capacité à opérer des continuités (thématique, conservation de l'enveloppe du bâti) et ruptures (arrêt de la production, nouvelles fonctionnalités) montre bien que des approches complexes, combinant plusieurs strates de temps peuvent être opérantes dès l'école. Cela se rapproche de la remise en perspective du temps du carbone dans l'anthropocène effectuée par BensaudeVincent et Loeve (2018, p. 375) « Au lieu de penser chaque être naturel ou artificiel comme immergé dans un temps unique - le temps universel de la physique - le carbone nous oblige à reconnaitre et à prendre en compte la diversité des figures de temps (linéaire, cyclique, feuilleté, etc.), bref à affronter la polychronie ».

Tout aménagement du territoire, plus spécialement lorsqu'il est question de paysage ou de terrain, procède d'une logique de conscientisation par le processus débutant par une perception, mobilisant un champ référentiel, puis aboutissant à une représentation (Bédart, 2012). L'approche sémiotique peircienne insiste sur la place pivot du champ référentiel, et le désigne par interprétant, pour produire de la signification. C'est à partir de celui-ci qu'un réseau de signification et d'interprétation sera possible et discuté collectivement. Concernant les trois projets souhaitant une rupture totale avec le territoire (changement de type transformation), le passé devient insignifiant et dans les discussions et les arguments de ces élèves le passé n'a pas de mots, pas d'existence possible. Le temps est réduit à un présent sans borne, assez proche des évolutions des régimes d'historicité proposées par Hartog (2003).

\subsection{Territoire de demain entre créativité rationnelle et trame narrative}

Les créativités mobilisées lors de ces travaux, selon la typologie de Joas (1999) se situeraient davantage entre les catégories primaire (représenter, imaginer) et secondaire (imaginer des solutions techniques, rationnelles). La dimension architecturale des projets se prête bien à ce type de mise en jeu cognitive et a été également l'occasion de mobiliser des savoirs techniques précis afin d'aboutir à des projets de requalifications de lieux. Ces solutions techniques apparaissent intégrer de multiples contraintes (volume des bâtiments, hauteur, matériaux) et les usages comme l'ascenseur extérieur du musée du terril. Elles sont le fruit d'une créativité rationnelle mais signifiant également une technicité inventive entre interprétation et appropriation, selon les registres de Martinand (1994).

La présence de signes graphiques liés à la mise en scène (disposition de figures humaines dans des espaces soigneusement choisis, juxtaposition de dessins façon BD, rajouts de mots, phrases ou détails horaires) inscrit le dessin dans les prémisses d'une trame narrative. Celle-ci signifie que les élèves se sont déportés vers la fonctionnalité de leur projet. Par l'inscription dans un quotidien et un tissu social ceci témoigne d'un début d'appropriation de la notion "d'usage », utilisée dans de nombreux contextes (architecture, sociologie, urbanisme, géographie...). Ce n'est pas un hasard si ces trames narratives apparaissent dans des projets liés au patrimoine. Di Méo (2008) constate que « le patrimoine est un 
discours, il participe d'un principe narratif (il a donc besoin de narrateurs) qui raconte les mythes originels, qui décrit les épopées fondatrices et les grands moments historiques d'un groupe ou d'un territoire. Il confère à toute réalité sociale une consistance temporelle (durée) et spatiale ». Di Méo (2008) conclut par le fait que ces fictions invitent à se projeter vers l'avenir et à formuler un projet collectif.

\section{Conclusion}

Au cours de ces investigations multiréférentielles, la mise en projet a facilité la rencontre d'enjeux complémentaires à travers une série de questionnements : Que mettre en patrimoine ? Comment réaliser un projet dans le futur? La diversité des projets au niveau du rendu graphique comme au niveau des idées témoigne d'un réel engagement dans un dispositif complexe et exigeant. La sollicitation de l'imagination et de la créativité a permis aux élèves d'entrer dans des processus d'écriture et de réécriture, se confrontant avec le réel, par le biais de ces requalifications d'espace en relation directe avec la notion de «territoire palimpseste » qui ne «cesse d'être raturé et récrire en permanence constituant un vieux grimoire des sols » (Corboz, 1983). Ce processus d'écriture et de réécriture a en outre permis la figuration d'évolutions, l'émergence de nouvelles formes, et rendu très concrètes les différentes formes de changements souhaitées par les élèves. Ceux-ci ont privilégié un changement de type métamorphose, permettant de mettre en valeur un lien avec le passé tout en projetant des aménagements vers l'avenir. Le fait de questionner les liens profonds entre temps et espaces via le patrimoine, amène à faire le constat de temporalités plus imbriquées les unes dans les autres, voir superposées que ne le pensaient les élèves. Une approche sociologique du territoire développée par Lahire (2012) en propose trois formes : territoires prescrits (institutionnels), territoires vécus (territoires d'actions) et territoires rêvés (territoires symboliques). Et il y a sans doute un peu de rêves dans la formule «territoire de demain » qui qualifie l'ensemble des projets conçus par les élèves. Le fil du patrimoine dans ce dispositif agrège et met en tension entre elles des dimensions hétérogènes de l'éducation au développement durable. L'engagement citoyen, la mobilisation de différents types de savoirs, la prise en compte du futur et la complexité se trouvent intimement intégrés dans une finalité qui les transcende.

\section{Bibliographie}

ABERNOT Y. \& BEDIN V. Changement d'évaluation. Evaluation du changement. Questions Vives N²3, 2015. DOI : 10.4000/questionsvives.1705

ARDOINO J., "L'approche multiréférentielle (plurielle) des situations éducatives et formatives ", Pratiques de FormationAnalyses, $\mathrm{n}^{\circ}$ 25-26, 13 p, tiré de http://docplayer.fr/21074296-L-approche-multireferentielle-plurielle-des-situationseducatives-et-formatives.html (consulté le 5 janvier 2018), 1993.

ARENDT H., La crise de la culture. Huit exercices de pensée politique, Paris, Gallimard, 1972.

AUDIGIER F., Éducation en vue du développement durable et didactiques, les cahiers des sciences de l'éducation-Université de Genève, N¹30, p. 47-71, 2011.

AUTRAN J., LOCHARD T. \& MONTEAU R., "L'exploitation dans le bassin minier de Provence, Quartiers, puits et galeries ", Travaux de l'observatoire Hommes milieux du Bassin Minier de Provence », CNRS, ISSN 2114-8147, 2013.

BARTHES A., BLANC-MAXIMIN S., ALPE Y. M. \& FLORO M., L'éducation au patrimoine : pourvoyeuse de savoirs et/ou au service des territoires ? Colloque international « les éducations à, un levier de transformation du système éducatif »1719 Novembre, Rouen (actes en ligne : https://halshs.archives-ouvertes.fr/halshs-01183403), 2014.

BARTHES A., Education au patrimoine in «Dictionnaire critique des enjeux et concepts des « éducations à »» dir. BARTHES A., LANGE J.M., et TUTIAUX-GUILLON N., Paris - L'Harmattan, 2017. 630 p., p182-190.

BATEMAN D., Transforming teachers' temporalities: Futures in an Australian classroom. Futures, 44, p. 14-23, 2012. 
BEDART M., Le projet de paysage ou l'opportunité d'un renouveau paradigmatique et identitaire grâce à un imaginaire fédérateur, instituant heuristique, in « L'imaginaire géographique : Perspectives, pratiques et devenirs. " Dir. Bédard M., Augustin J.-P. et Desnoilles R., Presse universitaires du Québec, Québec 2012. 376 p., p. 43-55, 2012.

BENSAUDE-VINCENT B. \& LOEVE S., Penser carbone, in " Penser l'anthropocène », Dir. Beau R. et Larrère C., Paris - Press de Sciences Po, 2018. 545p, p 364-380.

BERGSON H., La perception du changement, in La pensée et le mouvant, PUF, (79 ${ }^{\text {ème }}$ ed.), 1969.

BONNEUIL C. \& FRESSOZ J.-C., L'évènement Anthropocène, La Terre, l'histoire et nous. Paris - Seuil, (2eme éd.), 2016.

BOUGNOUX D. \& DEBRAY R., L'immuable et le mutant, Médium 35, 3-5. DOI 10.3917/mediu.035.0003, 2013.

BRONCKART J.P. «L'activité langagière, la langue et le signe, comme organisateurs du développement humain », in Langage \& société, vol. 2007/3-4, n 121-122, p. 57-68, 2007.

BROUSSAL D., Recherche-Intervention et accompagnement du changement en éducation: vers une approche contre culturelle de l'émancipation. Habilitation à Diriger des Recherches, Université Toulouse Jean Jaurés, 2017. https://hal.archives-ouvertes.fr/tel-01536098

COLLIGNON B., « Expériences et savoirs géographiques », Sciences Humaines, N¹37, p. 24-26, 2003.

Convention-cadre du Conseil de l'Europe sur la valeur du patrimoine culturel pour la société, Faro, 27.X.2005, https://rm.coe.int/1680083748, 2005.

CORBOZ A., Le territoire comme palimpseste, Diogène, 121, p.14-35, 1983.

DAVALLON J., A propos des régimes de patrimonialisation: enjeux et questions. Patrimonializao e sustentabilidade do patrimonio : reflexao et prospectiva, Nov 2014, Lisboa, Portugal <halshs-01123906>, 2014.

DESCOLA P., Par-delà nature et culture, Paris - Gallimard, 2005.

DEWEY J., Démocratie et éducation. Suivi de Expérience et éducation. Paris, France : Armand Colin. (Ouvrage original publié en 1916 sous le titre Democracy and Education. An Introduction to the Philosophy of Education), 2011.

DI MEO G., Processus de patrimonalisation et construction des territoires. Colloque " Patrimoine et industries en Poitou Charentes : connaître pour valoriser ", sep 2007, Poitiers Châtellerault, Geste Editions, p. 87-109. <halshs-00281934>, 2008.

DOREL-FERRE G., Identifier, inventorier, classer in Historiens et Géographes-spécial « Patrimoine industriel », N³98, p.115145, 2007.

GARFINCKEL H., Recherches en ethnométhodologie, édité et introduit par Barthélémy M. et Quéré L., Paris - PUF, 2007.

GODARD O., Environnement, modes de coordination et système de légitimité : analyse de la catégorie patrimoine naturel, Revue économique, vol 41, N², p. 215-241, 1990.

GOUGH N., Futures in Australian education-tacit, token and taken for granted, Futures 22(3), p. 298-310, 1990.

GOUGH N., Can we escape the program? Inventing possible impossible futures in/for Australian educational research. The Australian Educational Researcher, 37(4), p. 9-42, 2010.

HANCOCK T., Planning and creating healthy and sustainable cities: The challenge for the 21 st century. In C. Price et A. Tsouros (dir.), Our cities, our future: Policies and action plans for health and sustainable development. Copenhagen : World Health Organization, p. 6-17, 1996.

HARTOG F., Régimes d'historicité, présentisme et expérience du temps. Paris Le Seuil, 2003.

HICKS D. \& HOLDEN C., Remembering the future: what do children think? Environmental Education Research, 13(4), p. 501512, 2007.

JANNER-RAIMONDI M. \& TAVIGNOT P., La notion de changement en éducation et formation : entre approche plurielle et regards croisés, in J.L. RINAUDO et TAVIGNOT P., Le changement à l'école. Sources, tensions, effets. Paris, L'Harmattan, p. 15-40, 2016.

JULIEN M.-P., CHALMEAU R., VERGNOLLE MAINAR C., LENA J.-Y. \& CALVET A. Concevoir le futur d'un territoire dans une perspective d'éducation au développement durable, Vertigo, 14-1, 16 p., 2014. [http://vertigo.revues.org/14690] 
JULIEN M.-P., CHALMEAU R., VERGNOLLE MAINAR C. \& LENA J.-Y., An innovative framework for encouraging future thinking in ESD: a case study in a French school. Futures, 101, p. 26-35, 2018. [https://doi.org/10.1016/j.futures.2018.04.012]

JOAS H., la créativité de l'agir, Paris-Cerf. (Edition Fr), 1999.

JOLLIVET M. (dir) , Le développement durable de l'utopie au concept, de nouveaux chantiers pour la recherche. Paris Elsevier SAS, 2001.

JOLLIVET M., Éléments de théorie pour une recherche interdisciplinaire sur les interfaces Nature/société. Prologue dans Hervé D. et Laloë F. (dir) Modélisation de l'environnement entre nature et sociétés. Paris Quae, 2009.

LAHIRE B., Monde pluriel. Penser l'unité des sciences sociales. Paris : PUF, 2012.

LANGE J.-M. \& VICTOR P., Didactique curriculaire et éducation à... la santé, l'environnement et au développement durable : quelles questions, quels repères ? Didaskalia, 28, p. 85-100, 2006.

LANGE J.-M. \& MARTINAND J.-L., Curriculum de l'EDD : principes de conception et d'élaboration. In Colloque International "Education au développement durable et à la biodiversité : concepts, questions vives, outils et pratiques", Digne les Bains, France. pp.118-136, INTI-International Network of Territorial Intelligence. <halshs- 00958229>, 2010.

LANGE J.-M., Curriculum possible de l'Education au développement durable : entre actions de participation et investigations multiréférentielles d'enjeux. Education relative à l'environnement, nº 11, p. 41-60, 2013.

LATOUR B., La science en action, introduction à la sociologie des sciences. Paris : La Découverte (2eme Ed.), 2005.

LENA J.-Y., JULIEN M.-P., CHALMEAU R., CALVET A. \& VERGNOLLE-MAINAR C., Les ressources en eau dans l'environnement de proximité : des chercheurs dans la classe. RDST (Recherches en didactique des Sciences et des Technologies) $N^{\circ} 13, p$. 133-160, 2016.

LENIAUD J.-M., L'utopie française. Essai sur le patrimoine, Paris, Éditions Mengès, 1992.

MAPPIN M. \& JONHNSON E., Changing perspectives of ecology and education in environmental education. In, Johnson, E. and Mappin, M. (Eds.) Environmental Education and Advocacy : Changing perspectives of ecology and education, p. 1-28. Cambridge: Cambridge University Press, 2005.

MARTINAND J.-L, La didactique des sciences et de la technologie et la formation des enseignants. Aster, 19, p. 61-75, 1994.

MEAD G.H., L'esprit, le soi et la société. Traduction et introduction Céfaï D. et Quéré L., Paris - PUF, (1934, 1ere édition américaine). 436 p, 2006.

MICOUD A., Des patrimoines aux territoires durables. Ethnologie et écologie dans les campagnes françaises, Ethnologie française 2004/1 (Vol. 34), p. 13-22. DOI 10.3917/ethn.041.0013, 2004.

MICOUD A., La patrimonialisation ou comment redire ce nous relie (un point de vue sociologique) in Barrère C. et al (dir.) Réinventer le patrimoine. De la culture à l'économie : une nouvelle pensée du patrimoine ? Paris - L'Harmattan, p. 81107, 2005.

NIEDDU M., PETIT O. \& VIVIEN F.-D., Identités, patrimoines collectifs et développement soutenable, in Développement durable et territoires-Dossier 12 | 2009, mis en ligne le 16 avril 2009, consulté le 30 septembre 2016. URL : http:// developpementdurable.revues.org/8126, 2009.

PEIRCE C.S., Ecrits sur le signe (traduits par Deledalle G.), Paris-Seuil, 1978.

PESCE S., "Innovation et Sédimentation dans le langage scolaire : Créativité Linguistique et Fabrique de l'Institution ", Revue Penser l'Éducation, 23, p. 89-100, 2008.

PICHOT A., Petite phénoménologie de la connaissance - Paris, Aubier, 1991, 222 p., 1991.

PRUNEAU D. et Desjardins G., "L'éducation au développement urbain : Quelle éducation pour quels développements ? " Education relative à l'environnement, Vol. 4, p. 97-111, 2003.

ROSS A., Curriculum: construction and critique. London and New York : Routledge Falmer, 2000.

SAINT-JEAN M. \& SEDDAOUI F., Le concept de « développement » en question dans l'approche des différents niveaux de changements. in «Conduite et accompagnement du changement. Contribution des sciences de l'éducation »V. BEDIN, Paris - L’Harmattan, 2013, p179-191. 
SERRES M., Le Tiers-Instruit. Paris : F. Bourin, 1991.

TORNATORE J.-L., "L'esprit de patrimoine », Terrain, 55, 106-127 | consulté le 10 février 2018. URL : http://journals.openedition.org/terrain/14084; DOI : 10.4000/terrain.14084, 2010.

TOUBIN M., LHOMME S., DIAB Y., SERRE D. et LAGANIER R., La Résilience urbaine : un nouveau concept opérationnel vecteur de durabilité urbaine ? Développement durable et territoires, Vol. 3, $n^{\circ} 1$ | Mai 2012, URL : http://developpementdurable.revues.org/9208, 2012.

TUTIAUX-GUILLON N., Education au patrimoine in «Dictionnaire critique des enjeux et concepts des « éducations à » » dir. BARTHES A., LANGE J.M., et TUTIAUX-GUILLON N., Paris - L'Harmattan, 630 p., p. 191-193, 2017.

UNESCO, Feuille de route pour la mise en œuvre du programme d'action globale pour l'EDD, http://unesdoc.unesco.org/images/0023/002305/230514f.pdf, 2014.

VERGNOLLE MAINAR C., JULIEN M.-P., CHALMEAU R., CALVET A., et LENA J.-Y., " Recherches collaboratives » en éducation à l'environnement et au développement durable: l'enjeu de la modélisation de l'ingénierie éducative, pour une transférabilité d'un territoire à un autre, Éducation relative à l'environnement, Volume 13 - 1 | consulté le 14 mars 2017. URL : http://ere.revues.org/333, 2016.

VERGNOLLE MAINAR C., CALVET A., EYCHENNE L., MARQUE N., MICHINEAU D., et THOUZET A., " Regards disciplinaires croisés sur les paysages ordinaires de proximité : un enjeu pour enrichir le lien des élèves au territoire où ils habitent ", Education relative à l'environnement, $\mathrm{n}^{\circ}$ 10, p. 223-230, 2012. 


\section{Annexe 1}

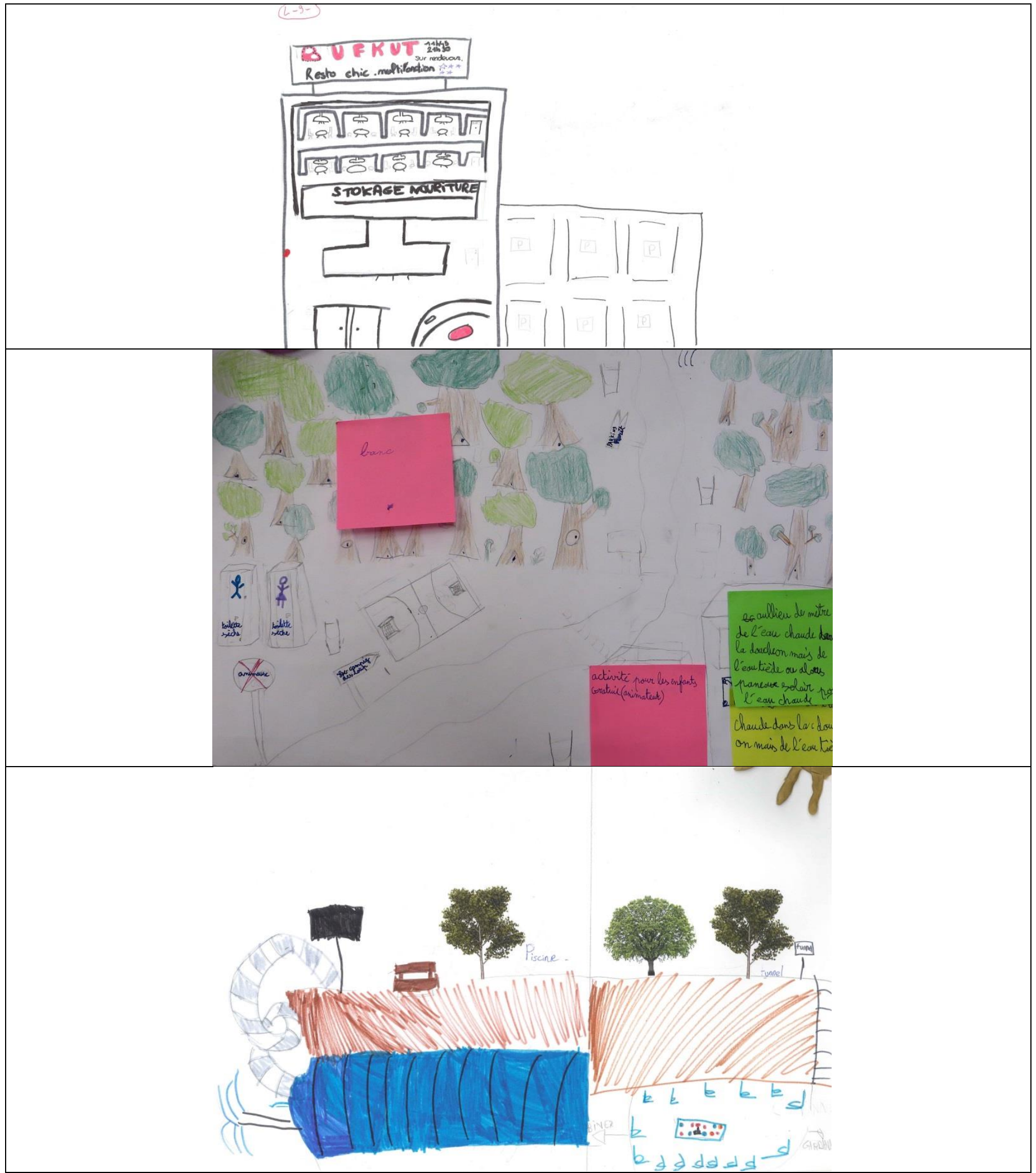

Exemples de projets : en haut : "Restau Europstar », au milieu " le camping des loups », en bas "Piscine sur le terril avec liaison souterraine avec Gardanne ». 


\section{Annexe 2}

\begin{tabular}{|c|c|c|c|c|c|c|}
\hline Qui & Nom des projets & Lieux & Signes princ. & interprétan & Patrimoine & Types de Chgt \\
\hline$A$ & $\begin{array}{c}\text { Gardanne biver dans le } \\
\text { futur }\end{array}$ & Biver & Tout changer - Tour & $\begin{array}{l}\text { Le tout } \\
\text { urbain }\end{array}$ & Peu & Transformation \\
\hline B & Micromania & Altéo & acheter / vendre & Commerce & Rien & Métamorphose \\
\hline $\mathrm{CJ}$ & Chadeux of evil & Forêt ou ville & la ville parc de loisir & Loisir & Rien & Transformation \\
\hline D & $\begin{array}{c}\text { Parc aqua. Téléphérique } \\
\text { souterain }\end{array}$ & Puits Gérard & usage du ss sol & Loisir & Bâti Requal. & Métamorphose \\
\hline KG & Piscine & Ferme Fabres & plan de masse & Loisir & Bâti Requal. & Métamorphose \\
\hline $\mathrm{H}$ & Go sport & Altéo & acheter / vendre & Commerce & Rien & Métamorphose \\
\hline I & Salle de sport & Puits Gérard & aéré - extérieurs & Loisir & peu & Métamorphose \\
\hline $\mathrm{L}$ & Restau Europstar & Altéo & projet intégré & Loisir com & Bâti Requal. & Métamorphose \\
\hline NE & Salle de sport & Maison ruine & & Loisir & Bâti Requal. & Transformation \\
\hline $\mathrm{O}$ & Pistes vélo et motos & Biver & s'amuser & Loisir & peu & Métamorphose \\
\hline PF & Camping des loups & Ferme & Camping « écolo » & Loisir & Forêt & Modifications \\
\hline Q & $\begin{array}{l}\text { Bâtiment omnisport + } \\
\text { restaurant }\end{array}$ & Puits Gérard & douches $\rightarrow$ restau & Loisir & Bâti Requal & Métamorphose \\
\hline $\mathrm{R}$ & Musée de la mine & Terril & & Cult. Mémo & $\mathrm{R}$ - construit & Métamorphose \\
\hline $\mathrm{S}$ & Musée de la mine & Puits Gérard & $\begin{array}{l}\text { Détails de } \\
\text { réamména. }\end{array}$ & Cult. Mémo & Bâti R & Métamorphose \\
\hline $\mathrm{T}$ & Centre commercial & Altéo & Enseignes & Commerce & Rien & Métamorphose \\
\hline UW & Musée Saint Jean & Puits Gérard & & Cult. Mémo & Bâti Requal. & Métamorphose \\
\hline V & Tout réorganisé & Toute la ville & La ville parc de loisir & Le tout urbain & Rien & Transformation \\
\hline$x$ & L'alumine & Altéo & intégré - recherche & Hybride & Bâti Requal. & Métamorphose \\
\hline ZB & Musée & Non précisé & Vision en 3D & Loisir & Bâti Requal. & Modification \\
\hline
\end{tabular}

Tableau synthétique des données sémiotiques et sémantiques de l'ensemble des projets (il est à noter que les lignes LTBH constituent 4 esquisses d'un même projet commun nommé « Europe star »). 


\section{Annexe 3. Avant - Après}

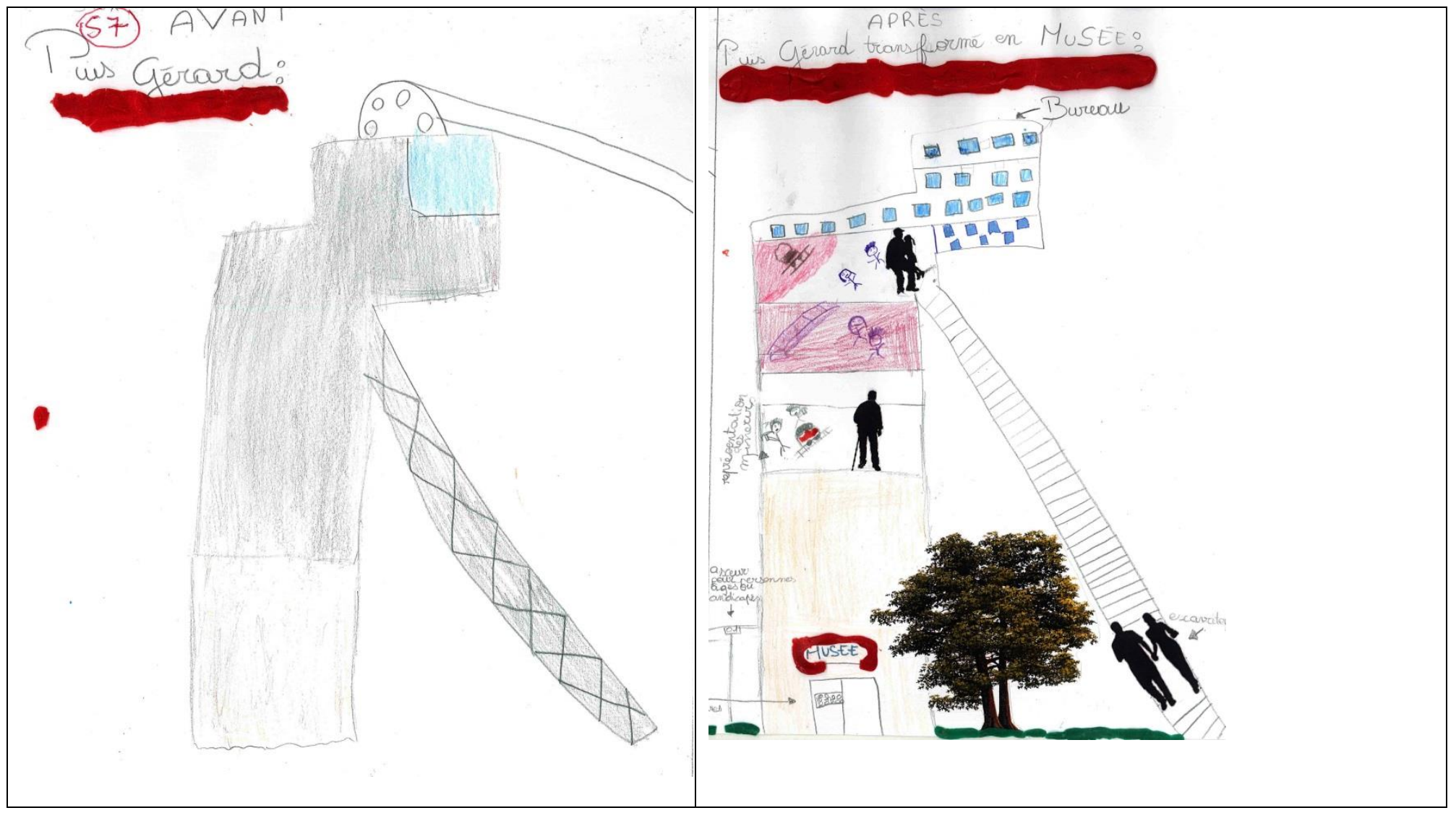

